\title{
Visibility in Semi-Convex Spaces ${ }^{\ddagger}$
}

\author{
Sven Schuierer* and Derick Wood ${ }^{\dagger}$ \\ Technical Report HKUST-CS95-39 \\ June 1995
}

\author{
*Institut für Informatik \\ Universität Freiburg \\ Rheinstr. 10-12 \\ D-79104 Freiburg \\ Germany \\ ${ }^{\dagger}$ Department of Computer Science \\ Hong Kong University of Science \& Technology \\ Clear Water Bay, Kowloon \\ Hong Kong
}

\begin{abstract}
We introduce the notion of a semi-convex space as a unifying framework for the treatment of various notions of convexity in the plane. Semi-convex spaces are a generalization of convexity spaces that are more appropriate for investigating issues of visibility. We define the notion of visibility within the general framework of semi-convex spaces, and investigate the relationship between visibility, kernels, and skulls. We prove the Kernel Theorem and the Cover Kernel Theorem, both of which relate kernels and skulls. Based on these results for semi-convex spaces we prove a theorem about metrics in the plane and demonstrate the utility of our theory with two examples of semi-convex spaces based on geodesic convexity and staircase convexity.
\end{abstract}

\footnotetext{
\$This research was supported by grants from the Natural Sciences and Engineering Research Council of Canada and by a grant from the Information Technolow Research Centre of Ontario.

The Hong Kong Technical Report Series University of Science \& Technology Department of Computer Science
} 


\title{
Visibility in Semi-Convex Spaces*
}

\author{
Sven Schuierer ${ }^{\dagger} \quad$ Derick Wood ${ }^{\ddagger}$
}

\begin{abstract}
We introduce the notion of a semi-convex space as a unifying framework for the treatment of various notions of convexity in the plane. Semi-convex spaces are a generalization of convexity spaces that are more appropriate for investigating issues of visibility. We define the notion of visibility within the general framework of semi-convex spaces, and investigate the relationship between visibility, kernels, and skulls. We prove the Kernel Theorem and the Cover Kernel Theorem, both of which relate kernels and skulls. Based on these results for semi-convex spaces we prove a theorem about metrics in the plane and demonstrate the utility of our theory with two examples of semi-convex spaces based on geodesic convexity and staircase convexity.
\end{abstract}

\section{Introduction}

The concept of visibility plays an important role in Computational Geometry. Its origins date back to 1973 when Victor Klee posed the now famous art gallery problem where he asked for the minimum number of guards that are always sufficient to guard an art gallery consisting of $n$ walls. Since then numerous visibility problems have been considered and a wealth of results has been obtained. Usually two points are defined to be visible to each other if the line segment that is spanned by the points does not intersect the interior of an obstacle. But apart from this definition several other notions of visibility have been investigated in recent years:

\footnotetext{
${ }^{*}$ This work was supported under grants from the Natural Sciences and Engineering Research Council of Canada and from the Information Technology Research Centre of Ontario.

${ }^{\dagger}$ Institut für Informatik, Universität Freiburg, Rheinstr. 10-12, D-79104 Freiburg, Germany; email: schuierer@informatik.uni-freiburg.de

${ }^{\ddagger}$ Department of Computer Science, Hong Kong University of Science \& Technology, Clear Water Bay, Kowloon, Hong Kong; e-mail: dwood@cs.ust.hk.
} 
staircase visibility [Bre94a, Bre94b, CR89, MRS88], rectangular visibility [Kei86, MOW87, OW88] and periscope visibility [GN90].

In this paper we consider several definitions of visibility and propose a framework in which many of the structural aspects of "non-standard" definitions of visibility can be treated in a uniform way. In order to do so, we employ an axiomatic approach based on convexity spaces. A convexity space is a tuple $\left(\mathbf{X}, \mathcal{C}_{\mathbf{X}}\right)$, where $\mathcal{C}_{\mathbf{X}}$ is the family of "convex sets" of the set $\mathbf{X}$. Convexity spaces represent an abstraction of the structure of convex sets in Euclidean space. Leaving aside all topological concerns, we require convex sets to be closed under intersection, thus capturing their lattice-theoretic and algebraic properties. It is not surprising that such a general concept has arisen in many different contexts and has led to names such as convexity spaces, convexity structures, or algebraic closure systems.

Convexity in this context is understood to be a property that depends solely on membership in $\mathcal{C}_{\mathbf{X}}$. Given $\mathcal{C}_{\mathbf{X}}$ we are led to the notion of the $\mathcal{C}_{\mathbf{X}}-$ hull of a set. Two points $p$ and $q$ in a set $\mathbf{S}$ are said to see each other if the $\mathcal{C}_{\mathbf{X}^{-}}$hull of $p$ and $q$ is contained in $\mathbf{S}$. By imposing various conditions on the sets in $\mathcal{C}_{\mathbf{X}}$ we can investigate some of the structural properties of the kernel of a set; in particular, we are able to characterize the convexity spaces for which the kernel can be obtained as the intersection of the family of maximal convex sets.

The original motivation for an abstract treatment of convexity is to investigate the relationships between the Helly, Radon, and Carathéodory numbers in an axiomatic setting [DRS81, Lev51, KW71, Sie77, Sie82]. The observation that many convexity spaces can be decomposed into simpler components has led to a second line of investigation. Its main goal is to deduce properties of the convexity space from those of its components [Deg79, Eck68, RW89, Sie81]. Many of the results obtained in this context can be found in the monograph by van de Vel [vdV93].

Our original motivation for an abstract treatment of visibility arose from our experience (shared with Greg Rawlins [Raw87]) of kernel-computation algorithms for polygons. Essentially, such algorithms compute a kernel as the intersection of the halfplanes defined by the edges of a polygon. Since each edge determines one skull (for many visibility notions), we have an intuitive basis for the Kernel Theorem (see Section 3 ) that relates skulls and kernels. Moreover, when there are finitely many skulls, the relationship suggests a kernel-computation algorithm. Although it may not be, however, the most efficient one. Surprisingly little attention has been paid in the theory of convexity spaces to problems that involve visibility, although visibility is a well 
studied concept in the context of real vector spaces [Bre82, Tor88, Val65].

As it turns out there is a variant of convexity spaces that is especially suited to treat questions that arise in the context of visibility. We call this variant semi-convex spaces.

The rest of the paper is organized as follows. In Section 2, we introduce the concept of semi-convex spaces and look at the notion of visibility in this abstract framework. This is followed by Section 3, the main section of this paper, with the presentation of our results on kernels and skulls in semi-convex spaces. It culminates in the proofs of the Kernel Theorem and the Cover Kernel Theorem. In Section 4 we study different methods of constructing semi-convex spaces and prove a general result about semiconvex spaces induced by metrics in the plane. Finally, we apply our results to a number of examples of semi-convex spaces in Section 5.

\section{Semi-convex Spaces and Visibility}

In this section we provide the definitions upon which we base our investigations of semi-convex spaces. We first define what we understand by a semi-convex space, establish some of the properties of the convex-hull operator in semi-convex spaces and abstract a suitable notion of visibility. Finally, we deal with skulls and aligned spaces.

\section{$2.1 \quad$ Semi-convex Spaces}

We base the following investigation on the concept of a semi-convex space which is a slight variant of a convexity space.

Definition 2.1 Let $\mathrm{X}$ be a set and $\mathcal{C}_{\mathrm{X}}$ be a collection of subsets of $\mathrm{X}$. Then, $\left(\mathrm{X}, \mathcal{C}_{\mathbf{X}}\right)$ is a semi-convex space if

1. $\emptyset$ is in $\mathcal{C}_{\mathbf{X}}$; and

2. for all $\mathcal{C} \subseteq \mathcal{C}_{\mathbf{X}}$, we have $\bigcap \mathcal{C} \in \mathcal{C}_{\mathbf{X}}$.

$\mathbf{X}$ is called the groundset of the convexity space and $\mathcal{C}_{\mathbf{X}}$ contains the "convex sets" of $\mathbf{X}$. In order to stress the analogy to Euclidean vector spaces we will call the elements of $\mathbf{X}$ points. Each set in $\mathcal{C}_{\mathbf{X}}$ is called $\mathcal{C}_{\mathbf{X}^{-}}$ convex (or convex for short if the convexity space is understood). ${ }^{1}$ So, the only characteristic required of convex sets is their closure under intersection.

\footnotetext{
${ }^{1}$ Note that the convexity of a set is solely determined by membership in $\mathcal{C}_{\mathbf{X}}$ and is not implied by any other property.
} 
The only difference in the definition of a semi-convex space from that of a convexity space is that in a convexity space $\mathbf{X}$ is also required to be an element of $\mathcal{C}_{\mathbf{X}}$. To see why we do not include $\mathbf{X}$ in $\mathcal{C}_{\mathbf{X}}$ consider a non-convex polygon $\mathbf{X}$ in the plane and let $\mathcal{C}_{\mathbf{X}}$ be the set of all convex subsets of $\mathbf{X}$. Clearly, $\left(\mathbf{X}, \mathcal{C}_{\mathbf{X}}\right)$ is a semi-convex space but not a convexity space though it captures the visibility structure of $\mathbf{X}$. Since we are interested in properties of $\mathbf{X}$ related to visibility, semi-convex spaces turn out to be the more useful setting. Indeed, from the viewpoint of visibility a convexity space is trivial since every point can see all the others.

Since convex sets are closed under intersection, we can define the convex hull of a set $\mathbf{Y} \subseteq \mathbf{X}$ in the semi-convex space $\left(\mathbf{X}, \mathcal{C}_{\mathbf{X}}\right)$ as usual by

$$
\mathcal{C}_{\mathbf{X}^{-}} \operatorname{hull}(\mathbf{Y})=\bigcap\left\{\mathbf{C} \in \mathcal{C}_{\mathbf{X}} \mid \mathbf{Y} \subseteq \mathbf{C}\right\} .
$$

But since we do not necessarily have that $\mathbf{X}$ is in $\mathcal{C}_{\mathbf{X}}$, it may be that $\left\{\mathbf{C} \in \mathcal{C}_{\mathbf{X}} \mid \mathbf{Y} \subseteq \mathbf{C}\right\}$ is empty. In topology one usually defines $\bigcap \emptyset=\mathbf{X}$ since it is assumed that any point $x$ satisfies $x \in \cap \emptyset$. We deviate from this tradition. If $\left\{\mathbf{C} \in \mathcal{C}_{\mathbf{X}} \mid \mathbf{Y} \subseteq \mathbf{C}\right\}=\emptyset$, then this means that $\mathbf{Y}$ contains points that cannot see each other in $\mathbf{X}$. The $\mathcal{C}_{\mathbf{X}}$ hull of $\mathbf{Y}$ does not exist and we define $\bigcap \emptyset=\emptyset$. Note that the converse also holds. We state it as an observation.

Observation 2.1 Let $\left(\mathrm{X}, \mathcal{C}_{\mathrm{X}}\right)$ be a semi-convex space and $\emptyset \neq \mathrm{Y} \subseteq \mathrm{X}$. Then, $\left\{\mathbf{C} \in \mathcal{C}_{\mathbf{X}} \mid \mathbf{Y} \subseteq \mathbf{C}\right\}=\emptyset$ if and only if $\mathcal{C}_{\mathbf{X}}-h$ ull $(\mathbf{Y})=\emptyset$.

\subsection{Visibility in Semi-convex Spaces}

If $\mathrm{Y}$ is a subset of $\mathrm{X}$, we define visibility in $\mathrm{Y}$ as follows.

Definition 2.2 Let $\left(\mathrm{X}, \mathcal{C}_{\mathrm{X}}\right)$ be a semi-convex space and $\mathrm{Y} \subseteq \mathrm{X}$. We say that two points $x$ and $y$ in $\mathbf{Y} \mathcal{C}_{\mathbf{Y}}$-see each other if $\mathcal{C}_{\mathbf{X}}-h u l l(\{x, y\}) \neq \emptyset$ and $\mathcal{C}_{\mathbf{X}}-\operatorname{hull}(\{x, y\}) \subseteq \mathbf{Y}$.

If we define $\mathcal{C}_{\mathbf{Y}}$ to be $\left\{\mathbf{C} \in \mathcal{C}_{\mathbf{X}} \mid \mathbf{C} \subseteq \mathbf{Y}\right\}$, then the above definition is equivalent to defining $x \quad \mathcal{C}_{\mathbf{Y}^{-}}$sees $y$ in $\mathbf{Y}$ iff $\mathcal{C}_{\mathbf{Y}^{-}} \operatorname{hull}(\{x, y\}) \neq \emptyset$.

Observe that $\mathcal{C}_{\mathbf{Y}^{-}}$sees is symmetric, but not necessarily reflexive or transitive. We have reflexivity, for all $\mathbf{Y} \subseteq \mathbf{X}$, if and only if all singletons in $\left(\mathbf{X}, \mathcal{C}_{\mathbf{X}}\right)$ are convex. We give a special name to semi-convex spaces that have this property.

Definition 2.3 A semi-convex space $\left(\mathbf{X}, \mathcal{C}_{\mathbf{X}}\right)$ is called simple if, for all $x \in$ $\mathbf{X}$, we have $\{x\} \in \mathcal{C}_{\mathbf{X}}$. 
Once having established a consistent definition of visibility it is easy to generalize the notion of a kernel for semi-convex spaces which we need in the remainder of this paper.

Definition 2.4 Let $\left(\mathrm{X}, \mathcal{C}_{\mathrm{X}}\right)$ be a semi-convex space and $\mathrm{Y} \subseteq \mathrm{X}$; then, $\mathcal{C}_{\mathbf{X}}-\operatorname{kernel}(\mathbf{Y})=\left\{y \in \mathbf{Y} \mid\right.$ for all $y^{\prime} \in \mathbf{Y}, y \mathcal{C}_{\mathbf{Y}}$-sees $\left.y^{\prime}\right\}$.

Because $\mathcal{C}_{\mathbf{X}^{-}}$hull is an expansive operator if it is nonempty, we deduce immediately that, for a nonempty convex set $\mathbf{C}$ and any two points $x$ and $y$ in $\mathbf{C}, \emptyset \neq \mathcal{C}_{\mathbf{X}^{-}} \operatorname{hull}(\{x, y\}) \subseteq \mathcal{C}_{\mathbf{X}^{-}} \operatorname{hull}(\mathbf{C})=\mathbf{C}$. Thus, for all points $x$ and $y$ in $\mathbf{C}$, we have $x \mathcal{C}_{\mathbf{C}^{-}}$sees $y$. The converse does not hold in general. This unexpected behaviour of semi-convex spaces leads to the concept of visibility convexity.

Definition 2.5 Let $\left(\mathrm{X}, \mathcal{C}_{\mathrm{X}}\right)$ be a semi-convex space and $\mathrm{C} \subseteq \mathrm{X}$. We say $\mathbf{C}$ is $\left(\mathcal{C}_{\mathbf{X}^{-}}\right)$visibility convex (all-seeing convex) if, for all $x, y \in \mathbf{C}$, we have $\mathcal{C}_{\mathbf{X}}-h$ ull $(\{x, y\}) \neq \emptyset$ and $\mathcal{C}_{\mathbf{X}}-h u l l(\{x, y\}) \subseteq \mathbf{C}$; in other words, $\mathcal{C}_{\mathbf{X}}-\operatorname{kernel}(\mathbf{C})=\mathbf{C}$.

Definition 2.6 A semi-convex space $\left(\mathrm{X}, \mathcal{C}_{\mathbf{X}}\right)$ is said to be complete if all visibility convex sets are convex.

Hence, in a complete semi-convex space, all-seeingness and convexity are equivalent notions. This property makes complete semi-convex spaces a natural setting for questions concerning visibility. Moreover, most of the semi-convex spaces that arise in practice are based on the definition of the convex hull of two points and extend this definition to a complete semiconvex space as, for instance, convexity in $I R^{n}$.

As another visibility-related concept we need the notion of a join.

Definition 2.7 Let $\left(\mathbf{X}, \mathcal{C}_{\mathbf{X}}\right)$ be a semi-convex space, $\mathbf{C} \in \mathcal{C}_{\mathbf{X}}$, and $x \in \mathbf{X}$, we define the $\mathcal{C}_{\mathbf{X}}$-join of $x$ and $\mathrm{C}$ by

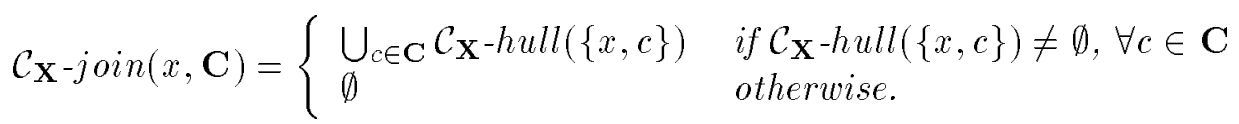

The join of a convex set $\mathbf{C}$ and a point $x$ consists, intuitively speaking, of all the line segments between $x$ and points $c$ in $\mathbf{C}$. It is easy to show that the join in the plane is always convex if we consider normal convexity. This, however, is not true for arbitrary semi-convex spaces which leads to our next definition. 
Definition 2.8 Let $\left(\mathrm{X}, \mathcal{C}_{\mathbf{X}}\right)$ be a semi-convex space. $\left(\mathrm{X}, \mathcal{C}_{\mathbf{X}}\right)$ is said to satisfy the join condition ${ }^{2}$ if, for all $x \in \mathbf{X}$ and $\mathbf{C} \in \mathcal{C}_{\mathbf{X}}$, we have $\mathcal{C}_{\mathbf{X}}-j \operatorname{oin}(x, \mathbf{C})$ is convex.

Note that if $\mathcal{C}_{\mathbf{X}^{-}} \operatorname{hull}(\{x\} \cup \mathbf{C}) \neq \emptyset$, then $\mathcal{C}_{\mathbf{X}^{-}} \operatorname{join}(x, \mathbf{C})$ is nonempty and the inclusion $\mathcal{C}_{\mathbf{X}^{-}}$join $(x, \mathbf{C}) \subseteq \mathcal{C}_{\mathbf{X}^{-}} h u l l(\{x\} \cup \mathbf{C})$ holds for any semi-convex space while the reverse inclusion only holds for semi-convex spaces that satisfy the join condition. This is stated in the following lemma.

Observation 2.2 Let $\left(\mathrm{X}, \mathcal{C}_{\mathrm{X}}\right)$ be a semi-convex space. $\left(\mathrm{X}, \mathcal{C}_{\mathrm{X}}\right)$ satisfies the join condition if and only if, for all $x \in \mathbf{X}$ and $\mathbf{C} \in \mathcal{C}_{\mathbf{X}} \backslash\{\emptyset\}$, we have that $\mathcal{C}_{\mathbf{X}}-\operatorname{hull}(\{x\} \cup \mathbf{C})=\mathcal{C}_{\mathbf{X}}-j \operatorname{oin}(x, \mathbf{C})$.

If we consider complete semi-convex spaces (which is what we do in most cases) the join condition can be reduced to the following three-point condition.

Theorem 2.3 If $\left(\mathrm{X}, \mathcal{C}_{\mathbf{X}}\right)$ is a complete semi-convex space, then the following two conditions are equivalent.

1. For all points $x, y$, and $z$ in $\mathbf{X}, \mathcal{C}_{\mathbf{X}}-j \operatorname{oin}\left(x, \mathcal{C}_{\mathbf{X}}-h u l l(\{y, z\})\right)$ is convex.

2. For all points $x$ in $\mathbf{X}$ and for all sets $\mathbf{C}$ in $\mathcal{C}_{\mathbf{X}}, \mathcal{C}_{\mathbf{X}}-j \operatorname{join}(x, \mathbf{C})$ is convex.

Proof: $1 \Rightarrow 2$. Consider some point $x$ in $\mathbf{X}$ and some $\mathbf{C}$ in $\mathcal{C}_{\mathbf{X}}$. We denote $\mathcal{C}_{\mathbf{X}^{-}}$join $(x, \mathbf{C})$ by $\mathbf{Q}$. We assume that $\mathbf{Q}$ is nonempty since if $\mathcal{C}_{\mathbf{X}^{-}}$join $(x, \mathbf{C})=\emptyset$, then $\mathcal{C}_{\mathbf{X}^{-}} j \operatorname{oin}(x, \mathbf{C})$ is convex and we are done. For every pair $y_{1}$ and $y_{2}$ of points in $\mathbf{Q}$, we have to show that $\mathcal{C}_{\mathbf{X}}-h u l l\left(\left\{y_{1}, y_{2}\right\}\right) \neq \emptyset$ and $\mathcal{C}_{\mathbf{X}}-h u l l\left(\left\{y_{1}, y_{2}\right\}\right) \subseteq \mathbf{Q}$. Because $\left(\mathbf{X}, \mathcal{C}_{\mathbf{X}}\right)$ is complete, this implies that $\mathbf{Q}$ is convex.

So let $y_{1}, y_{2} \in \mathbf{Q}$. Since $\mathbf{Q} \neq \emptyset$, there are two points $c_{1}$ and $c_{2}$ in $\mathbf{C}$ with $y_{i} \in \mathcal{C}_{\mathbf{X}}-h u l l\left(\left\{x, c_{i}\right\}\right)$, for $i=1,2$. Because $c_{1}$ and $c_{2}$ are in $\mathbf{C}$, we have that $\mathcal{C}_{\mathbf{X}^{-}} \operatorname{hull}\left(\left\{c_{1}, c_{2}\right\}\right)$ is nonempty and $\mathcal{C}_{\mathbf{X}^{-}} \operatorname{hull}\left(\left\{c_{1}, c_{2}\right\}\right) \subseteq \mathbf{C} \subseteq \mathbf{Q}$. Since $\mathbf{Q} \neq \emptyset$, we further have that $\mathcal{C}_{\mathbf{X}}-\operatorname{hull}(\{x, y\}) \neq \emptyset$, for any $y \in \mathcal{C}_{\mathbf{X}}-h u l l\left(\left\{c_{1}, c_{2}\right\}\right)$ and, therefore, $\mathcal{C}_{\mathbf{X}^{-}} \operatorname{join}\left(x, \mathcal{C}_{\mathbf{X}^{-}} \operatorname{hull}\left(\left\{c_{1}, c_{2}\right\}\right)\right)$ is nonempty and contains $y_{1}$ and $y_{2}$. But, $\mathcal{C}_{\mathbf{X}^{-}}$join $\left(x, \mathcal{C}_{\mathbf{X}^{-}} \operatorname{hull}\left(\left\{c_{1}, c_{2}\right\}\right)\right)$ is convex, by assumption; hence, we have that $\mathcal{C}_{\mathbf{X}^{-}} \operatorname{hull}\left(\left\{y_{1}, y_{2}\right\}\right) \subseteq \mathcal{C}_{\mathbf{X}^{-}} \operatorname{join}\left(x, \mathcal{C}_{\mathbf{X}^{-}} \operatorname{hull}\left(\left\{c_{1}, c_{2}\right\}\right)\right) \subseteq \mathcal{C}_{\mathbf{X}^{-}} \operatorname{join}(x, \mathbf{C})=$ $\mathbf{Q}$ and $\mathcal{C}_{\mathbf{X}^{-}} \operatorname{hull}\left(\left\{y_{1}, y_{2}\right\}\right) \neq \emptyset$.

$2 \Rightarrow 1$. Trivial.

\footnotetext{
${ }^{2}$ This is also called $\mathcal{C}_{\mathrm{X}}$-join commutativity [vdV93].
} 


\subsection{Skulls and Aligned Semi-convex spaces}

It is the main aim of this paper to relate the maximal convex subsets of a set (which we call "skulls") to its kernel.

Definition 2.9 Let $\left(\mathrm{X}, \mathcal{C}_{\mathbf{X}}\right)$ be a semi-convex space and $\mathrm{Y} \subseteq \mathrm{X}$. Then,

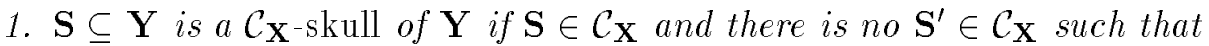
$\mathrm{S} \subset \mathrm{S}^{\prime} \subseteq \mathrm{Y}$.

2. We denote the family $\left\{\mathbf{S} \subseteq \mathbf{Y} \mid \mathbf{S}\right.$ is a $\mathcal{C}_{\mathbf{X}}-$ skull of $\left.\mathbf{Y}\right\}$ of all skulls in $\mathbf{Y}$ by $\mathcal{C}_{\mathbf{X}}-$ skulls $(\mathbf{Y})$.

Note that given a set $\mathbf{Y} \subseteq \mathbf{X}$ in a semi-convex space $\left(\mathbf{X}, \mathcal{C}_{\mathbf{X}}\right)$ and a convex subset $\mathbf{C}$ of $\mathbf{Y}$, there is not necessarily a skull that contains $\mathbf{C}$. This can be easily seen by considering the semi-convex space of closed intervals in $(0,1)$.

We say a semi-convex space is a space with skulls if, for all subsets $\mathbf{Y}$ of $\mathbf{X}$ and all convex sets $\mathbf{C} \subseteq \mathbf{Y}$, there is a skull $\mathbf{S} \in \mathcal{C}_{\mathbf{X}^{-}}$skulls $(\mathbf{Y})$ that contains $\mathbf{C}$. The above example suggests that we may also require that the union of nested chains ${ }^{3}$ of convex sets to be convex as well.

Definition 2.10 Let $\left(\mathrm{X}, \mathcal{C}_{\mathbf{X}}\right)$ be a semi-convex space. We call $\left(\mathrm{X}, \mathcal{C}_{\mathbf{X}}\right)$ an aligned (semi-convex) space if, for every nested chain $\mathcal{N} \subseteq \mathcal{C}_{\mathbf{X}}$, the union of $\mathcal{N}$ is also convex; that is, $\bigcup \mathcal{N} \in \mathcal{C}_{\mathbf{X}}$.

Aligned spaces are well studied objects in the literature [Ham63, Jam82, Sie84] but usually for quite different reasons than those stated here. The following lemma shows that the existence of skulls is, indeed, guaranteed by forcing the union of nested families to be convex.

Lemma 2.4 If $\left(\mathrm{X}, \mathcal{C}_{\mathrm{X}}\right)$ is an aligned space, then we have that, for all $\mathrm{Y} \subseteq$ $\mathrm{X}$ and for all $\mathbf{C} \in \mathcal{C}_{\mathbf{X}}$ with $\mathbf{C} \subseteq \mathbf{Y}$, there is a $\mathcal{C}_{\mathbf{X}}-$ skull $\mathbf{S}$ of $\mathbf{Y}$ that contains C.

Proof: To see this, take any $\mathbf{Y} \subseteq \mathbf{X}$ and $\mathbf{C}_{0} \in \mathcal{C}_{\mathbf{X}} \backslash\{\emptyset\}$ with $\mathbf{C}_{0} \subseteq \mathbf{Y}$.

Let $\mathcal{S}$ be the family of all convex sets in $\mathbf{Y}$ that contain $\mathbf{C}_{0}$. Note that $\mathcal{S}$ is nonempty. $\mathcal{S}$ is a partially-ordered set and any nested chain $\mathcal{N} \subseteq \mathcal{S}$ has an upper bound $\bigcup \mathcal{N} \in \mathcal{C}_{\mathbf{X}}$ with $\mathbf{C}_{0} \subseteq \bigcup \mathcal{N} \subseteq \mathbf{Y}$, since $\left(\mathbf{X}, \mathcal{C}_{\mathbf{X}}\right)$ is an aligned

\footnotetext{
${ }^{3}$ A nested chain $\mathcal{N}$ is a family of sets that is totally ordered with respect to inclusion.
} 
space. By Zorn's lemma, there is a maximal element $\mathbf{S}$ in $\mathcal{S}$; clearly then, $\mathbf{S}$ is a $\mathcal{C}_{\mathbf{X}^{-}}$skull of $\mathbf{Y}$ containing $\mathbf{C}$.

Another counter-intuitive observation is that $\mathcal{C}_{\mathbf{X}^{-}}$skulls $(\mathbf{Y})$ need not necessarily cover $\mathbf{Y}$, in the sense that $\bigcup \mathcal{C}_{\mathbf{X}^{-}}$skulls $(\mathbf{Y})$ is not necessarily $\mathbf{Y}$ itself, but only a subset. Whenever an aligned space is simple, however, we obtain a cover since every point is a convex subset and, therefore, there exists a maximal convex subset containing it.

We have introduced the notions of complete and aligned spaces above. Fortunately, there is a straightforward relationship between them.

Lemma 2.5 Let $\left(\mathbf{X}, \mathcal{C}_{\mathbf{X}}\right)$ be a semi-convex space. If $\left(\mathbf{X}, \mathcal{C}_{\mathbf{X}}\right)$ is complete, then $\left(\mathbf{X}, \mathcal{C}_{\mathbf{X}}\right)$ is an aligned space.

Proof: Let $\mathcal{N}$ be a nested chain of convex sets and let $\mathbf{C}=\bigcup \mathcal{N}$. Without loss of generality assume that $\mathbf{C} \neq \emptyset$. Further, let $x, y$ be arbitrary points in $\mathbf{C}$. It suffices to show that $x$ and $y$ see each other in $\mathbf{C}$ to prove that $\mathbf{C}$ is convex because $\left(\mathbf{X}, \mathcal{C}_{\mathbf{X}}\right)$ is complete. For $x$ and $y$ there are two convex sets $\mathbf{C}_{x}$ and $\mathbf{C}_{y}$ in $\mathcal{N}$ such that $x \in \mathbf{C}_{x}$ and $y \in \mathbf{C}_{y}$. Since $\mathcal{N}$ is a nested chain we can assume that $\mathbf{C}_{x} \subseteq \mathbf{C}_{y}$. So $\mathbf{C}_{x} \cup \mathbf{C}_{y}=\mathbf{C}_{y}$ is convex, and $\emptyset \neq \mathcal{C}_{\mathbf{X}}-h u l l(\{x, y\}) \subseteq \mathbf{C}_{y} \subseteq \mathbf{C}$.

So whenever we are dealing with complete semi-convex spaces Lemma 2.5 ensures that skulls automatically exist. There is also a (weak) converse that requires the join condition (see Theorem 2 in Kay and Womble [KW 71] for a different proof where aligned convexity spaces are considered).

Lemma 2.6 Let $\left(\mathrm{X}, \mathcal{C}_{\mathbf{X}}\right)$ be a semi-convex space with skulls. If $\left(\mathrm{X}, \mathcal{C}_{\mathbf{X}}\right)$ satisfies the join condition, then $\left(\mathrm{X}, \mathcal{C}_{\mathbf{X}}\right)$ is complete.

Proof: Consider a set $\mathbf{C} \subseteq \mathbf{X}$ which is visibility convex. We prove that $\mathbf{C}$ is convex, i.e., $\mathbf{C} \in \mathcal{C}_{\mathbf{X}}$. Since $\mathbf{C}$ is nonempty, it contains at least one point $x$, and because $\emptyset \neq \mathcal{C}_{\mathbf{X}}-h u l l(\{x, x\}) \subseteq \mathbf{C}$, by assumption, there is a $\mathcal{C}_{\mathbf{X}^{-}}$skull $\mathbf{S}_{x}$ in $\mathbf{C}$ with $x \in \mathbf{S}_{x}$. Now, take a point $y \in \mathbf{C}$; then, we have $\emptyset \neq \mathcal{C}_{\mathbf{X}}-h u l l(\{s, y\}) \subseteq \mathbf{C}$, for all points $s \in \mathbf{S}_{x}$, since $y$ can see all the points in $\mathbf{C}$. In other words, $\mathcal{C}_{\mathbf{X}^{-}}$join $\left(y, \mathbf{S}_{x}\right) \subseteq \mathbf{C}$.

But, $\mathcal{C}_{\mathbf{X}^{-}}$join $\left(y, \mathbf{S}_{x}\right)$ is nonempty since it is contained in $\mathbf{C}$ by assumption and convex. By the definition of a $\mathcal{C}_{\mathbf{X}^{-}}$skull this implies that $\mathcal{C}_{\mathbf{X}^{-}} \operatorname{join}\left(y, \mathbf{S}_{x}\right)=\mathbf{S}_{x}$. Thus, $y \in \mathbf{S}_{x}$ and $\mathbf{S}_{x}=\mathbf{C}$; therefore, $\mathbf{C}$ is convex. 


\section{The Kernel Theorem}

This section contains the main results of the paper. We state and prove the Kernel Theorem which gives a complete characterization of those semiconvex spaces for which the kernel of a set $\mathrm{Y}$ is given by the intersection of all skulls in $\mathbf{Y}$; hereby, we make crucial use of the concepts introduced so far. The proof of the theorem is partially due to Rawlins [Raw87, Theorem 6.3.1] who gives a proof of the "if" part in the case of convexity spaces under slightly stronger assumptions. Unfortunately, he fails to recognize that the existence of skulls has to be required and, hence, has no analog to Condition 1.

Theorem 3.1 (The Kernel Theorem) Let $\left(\mathrm{X}, \mathcal{C}_{\mathrm{X}}\right)$ be a semi-convex space. Then, we have, for all $\mathrm{Y} \subseteq \mathrm{X}$,

$$
\mathcal{C}_{\mathbf{X}}-\operatorname{kernel}(\mathbf{Y})=\bigcap \mathcal{C}_{\mathbf{X}}-\operatorname{skulls}(\mathbf{Y})
$$

if and only if either $\mathcal{C}_{\mathbf{X}}=\{\emptyset\}$ or the following three conditions hold:

1. $\left(\mathbf{X}, \mathcal{C}_{\mathbf{X}}\right)$ is a semi-convex space with skulls;

2. For all $x \in \mathbf{X}$ and for all $\mathbf{C} \in \mathcal{C}_{\mathbf{X}}, \mathcal{C}_{\mathbf{X}}-j \operatorname{oin}(x, \mathbf{C})$ is convex;

3. There exists a set $\mathbf{C}_{0} \in \mathcal{C}_{\mathbf{X}}$ such that, for all $x \in \mathbf{X}, \mathcal{C}_{\mathbf{X}}$-hull $(\{x\})=$ $\{x\} \cup \mathbf{C}_{0}$;

Proof: The claim clearly holds for $\mathcal{C}_{\mathbf{X}}=\{\emptyset\}$, so we immediately turn to the case $\mathcal{C}_{\mathbf{X}} \neq\{\emptyset\}$. Let $K=\mathcal{C}_{\mathbf{X}^{-}} \operatorname{kernel}(\mathbf{Y})$ and $I=\bigcap \mathcal{C}_{\mathbf{X}^{-}}$skulls $(\mathbf{Y})$.

if. We split the proof into two parts.

$K \subseteq I$. If $K=\emptyset$, this holds vacuously, so assume that $K \neq \emptyset$. Consider $x \in K$; we prove that $x \in I$. Let $\mathbf{S}$ be a skull in $\mathcal{C}_{\mathbf{X}^{-}}$skulls $(\mathbf{Y})$ and $s$ a point in $\mathbf{S}$; since $x \in \mathcal{C}_{\mathbf{X}^{-}} \operatorname{kernel}(\mathbf{Y})$, we have $x \mathcal{C}_{\mathbf{Y}^{-}}$sees $s$. Thus, $\mathcal{C}_{\mathbf{X}}$-hull $(\{x, s\})$ is nonempty and contained in $\mathbf{Y}$. Since $s$ is arbitrary, we have that $\mathbf{S} \subseteq \mathcal{C}_{\mathbf{X}^{-}}$join $(x, \mathbf{S})=\bigcup_{s \in \mathbf{S}} \mathcal{C}_{\mathbf{X}^{-}} h u l l(\{x, s\}) \subseteq$ $\mathbf{Y}$; furthermore, $\mathcal{C}_{\mathbf{X}^{-}}$join $(x, \mathbf{S})$ is convex, by assumption. But $\mathbf{S}$ is a maximal inscribed convex set of $\mathbf{Y}$; therefore, $\mathcal{C}_{\mathbf{X}^{-}}$join $(x, \mathbf{S})=\mathbf{S}$, $x \in \mathbf{S}$, and hence $x \in I$.

$I \subseteq K$. Again assume that $I \neq \emptyset$ and consider $x \in I$ and an arbitrary point $y \in \mathbf{Y}$. We have to show that $x \mathcal{C}_{\mathbf{Y}^{-}}$sees $y$. Since $\mathcal{C}_{\mathbf{X}^{-}}$hull $(\{x\})=\{x\} \cup$ 
$\mathbf{C}_{0} \subseteq I \subseteq \mathbf{Y}$ and $I$ is convex and nonempty, we have $\mathcal{C}_{\mathbf{X}}-h u l l(\{y\})=$ $\{y\} \cup \mathbf{C}_{0} \subseteq \mathbf{Y}$ by Condition 3. Furthermore, since $\mathcal{C}_{\mathbf{X}^{-}} h u l l(\{y\}) \subseteq \mathbf{Y}$, we know that there is an $\mathbf{S}_{y} \in \mathcal{C}_{\mathbf{X}^{-}}$skulls $(\mathbf{Y})$ with $\mathcal{C}_{\mathbf{X}^{-}}$hull $(\{y\}) \subseteq$ $\mathbf{S}_{y}$. Now $x \in I \subseteq \mathbf{S}_{y}$ and, thus, $\mathcal{C}_{\mathbf{X}}-h u l l(\{x, y\}) \subseteq \mathbf{S}_{y} \subseteq \mathbf{Y}$ and $\mathcal{C}_{\mathbf{X}^{-h u l l}}(\{x, y\}) \neq \emptyset$. Therefore, $x \mathcal{C}_{\mathbf{Y}^{-}}$sees $y$ and $x \in K$.

only if. Assume that $\mathcal{C}_{\mathbf{X}} \neq\{\emptyset\}$ and that, for all $\mathbf{Y} \subseteq \mathbf{X}$, we have that $\mathcal{C}_{\mathbf{X}}$-kernel $(\mathbf{Y})$ equals $\cap \mathcal{C}_{\mathbf{X}^{-}}$skulls $(\mathbf{Y})$. We prove that $\left(\bar{X}, \mathcal{C}_{\mathbf{X}}\right)$ satisfies the three conditions of the statement.

Condition 1: We have to show that skulls exist in $\left(\mathbf{X}, \mathcal{C}_{\mathbf{X}}\right)$. In order to do so we make use of Lemma 2.4 and show that $\left(\mathbf{X}, \mathcal{C}_{\mathbf{X}}\right)$ is aligned. For this let $\mathcal{N} \subseteq \mathcal{C}_{\mathbf{X}}$ be a nested chain of convex sets and let $\mathbf{Q}$ denote $\cup \mathcal{N}$. Consider $x, y \in \mathbf{Q}$. As in the proof of Lemma 2.5, we obtain a set $\mathbf{C} \in \mathcal{N}$ with $x, y \in \mathbf{C}$; thus $\mathcal{C}_{\mathbf{X}}-h u l l(\{x, y\}) \subseteq \mathbf{C} \subseteq \mathbf{Q}$ and $\mathcal{C}_{\mathbf{X}}-\operatorname{hull}(\{x, y\}) \neq \emptyset$. Hence, $\mathcal{C}_{\mathbf{X}}-\operatorname{kernel}(\mathbf{Q})=\mathbf{Q}$ and since $\mathcal{C}_{\mathbf{X}}$-kernel $(\mathbf{Q})$ is convex (it is the intersection of convex sets), we have $\mathbf{Q} \in \mathcal{C}_{\mathbf{X}}$

Condition 2: We now prove that the $\mathcal{C}_{\mathbf{X}^{-}}$join is convex. Consider an arbitrary point $x \in \mathbf{X}$ and an arbitrary $\mathbf{C} \in \mathcal{C}_{\mathbf{X}} \backslash\{\emptyset\}$; we show that $\mathcal{C}_{\mathbf{X}^{-}}$join $(x, \mathbf{C})$ which we denote by $\mathbf{Y}$ is convex. Without loss of generality assume that $\mathbf{Y} \neq \emptyset$. For all $y \in \mathbf{Y}$, we have $y \in \mathcal{C}_{\mathbf{X}}-h u l l(\{x, c\}) \neq$ $\emptyset$, for some $c \in \mathbf{C}$. But, this implies that $\mathcal{C}_{\mathbf{X}}-h u l l(\{x, y\}) \neq \emptyset$ and $\mathcal{C}_{\mathbf{X}}$ hull $(\{x, y\}) \subseteq \mathcal{C}_{\mathbf{X}^{-}} \operatorname{hull}(\{x, c\}) \subseteq \mathbf{Y}$; hence, $x \mathcal{C}_{\mathbf{Y}^{-}}$sees $y$. In other words, $x \in \mathcal{C}_{\mathbf{X}^{-}} \operatorname{kernel}(\mathbf{Y})$ and, thus, $x \in \bigcap \mathcal{C}_{\mathbf{X}^{-}}$skulls $(\mathbf{Y})$. Therefore, all skulls of $\mathbf{Y}$ contain $x$. Because $\left(\mathbf{X}, \mathcal{C}_{\mathbf{X}}\right)$ is an aligned space as we proved above, there is a skull $\mathbf{S}$ of $\mathbf{Y}$ that contains $\mathbf{C}$. $\mathbf{S}$ also contains $x$ so $\mathbf{Y}=\mathcal{C}_{\mathbf{X}^{-}} \operatorname{join}(x, \mathbf{C}) \subseteq \mathcal{C}_{\mathbf{X}^{-}} h u l l(\{x\} \cup \mathbf{C}) \subseteq \mathbf{S} \subseteq \mathbf{Y}$ and $\mathbf{Y}$ is convex.

Condition 3: First we prove that $x \in \mathcal{C}_{\mathbf{X}}$-hull $(\{x\})$, for all $x \in \mathbf{X}$. Assume the contrary; that is, there is an $x \in \mathbf{X}$ with $x \notin \mathcal{C}_{\mathbf{X}}-h u l l(\{x\})$ or, in other words, $x \notin \mathbf{C}$, for all $\mathbf{C} \in \mathcal{C}_{\mathbf{X}}$. Since $\mathcal{C}_{\mathbf{X}} \neq\{\emptyset\}$, there is a $\mathbf{C} \in \mathcal{C}_{\mathbf{X}}$ with $\mathbf{C} \neq \emptyset$. Let $\mathbf{Y}=\{x\} \cup \mathbf{C}$. Since $x$ is not contained in a convex set, $\mathbf{Y}$ is not in $\mathcal{C}_{\mathbf{X}}$ and, in particular, $\mathbf{Y} \neq \mathbf{C}$. Therefore, $\mathcal{C}_{\mathbf{X}^{-}}$skulls $(\mathbf{Y})=\{\mathbf{C}\}$ and $\bigcap \mathcal{C}_{\mathbf{X}^{-}}$skulls $(\mathbf{Y})=\mathbf{C}$. But no point in $\mathbf{Y}$ can see $x$ and, hence, $\mathcal{C}_{\mathbf{X}}-\operatorname{kernel}(\mathbf{Y})=\emptyset$ which contradicts the assumption that the kernel equals the intersection of the skulls.

Next we show that, for all $x, y \in \mathbf{X}$, we have $\mathcal{C}_{\mathbf{X}}-h u l l(\{y\}) \subseteq\{y\} \cup$ $\mathcal{C}_{\mathbf{X}}$-hull $(\{x\})$. Consider two arbitrary points $x$ and $y$ in $\mathbf{X}$ and let $\mathbf{Y}=$ $\mathcal{C}_{\mathbf{X}}-h u l l(\{x\}) \cup\{y\}$. We prove that $\mathcal{C}_{\mathbf{X}}-h u l l(\{y\}) \subseteq \mathbf{Y}$ by contradiction. 
Assume that $\mathcal{C}_{\mathbf{X}}-\operatorname{hull}(\{y\}) \nsubseteq \mathbf{Y}$. Then, for all $z \in \mathbf{Y}$, either $\mathcal{C}_{\mathbf{X}^{-}} \operatorname{hull}(\{y, z\}) \nsubseteq \mathbb{Y}$ or $\mathcal{C}_{\mathbf{X}}-\operatorname{hull}(\{y, z\})=\emptyset$ and, hence, $y$ is not visible from any point in $\mathbf{Y}$ (including itself). Thus, $\mathcal{C}_{\mathbf{X}}-\operatorname{kernel}(\mathbf{Y})=\emptyset$.

We have by similar reasoning that, for every $\mathbf{S} \in \mathcal{C}_{\mathbf{X}^{-}}$skulls $(\mathbf{Y}), y$ is not in $\mathbf{S}$. But since $\mathcal{C}_{\mathbf{X}^{-}}$hull $(\{x\}) \neq \emptyset, \mathcal{C}_{\mathbf{X}^{-}}$skulls $(\mathbf{Y})$ contain at least one skull $\mathbf{S}$ with $\mathbf{S} \supseteq \mathcal{C}_{\mathbf{X}}-h u l l(\{x\})$. Both statements together imply that $\mathbf{S}=\mathcal{C}_{\mathbf{X}}-$ hull $(\{x\})$ and $\mathcal{C}_{\mathbf{X}}-h u l l(\{x\})$ is the only skull of $\mathbf{Y}$. Thus, $\cap \mathcal{C}_{\mathbf{X}}-\operatorname{skulls}(\mathbf{Y})=\mathcal{C}_{\mathbf{X}}-\operatorname{hull}(\{x\}) \neq \emptyset$ and we have a contradiction; so $\mathcal{C}_{\mathbf{X}^{-}} h u l l(\{y\}) \subseteq \mathbf{Y}$.

Now let $\mathbf{C}_{0}=\bigcap_{x \in \mathbf{X}} \mathcal{C}_{\mathbf{X}}-h u l l(\{x\})$. Consider a point $y \in \mathbf{X}$. We have shown by the preceding arguments that $\mathcal{C}_{\mathbf{X}}-h u l l(\{y\}) \subseteq\{y\} \cup$ $\bigcap_{x \in \mathbf{X}} \mathcal{C}_{\mathbf{X}^{-}}$hull $(\{x\})=\{y\} \cup \mathbf{C}_{0}$. Since $\mathcal{C}_{\mathbf{X}^{-}}$hull $(\{y\})$ clearly contains $\mathbf{C}_{0}$ and also contains $y$ by the previous arguments, $\mathcal{C}_{\mathbf{X}}-h u l l(\{y\})=$ $\{y\} \cup \mathbf{C}_{0}$ as claimed.

As an immediate consequence we get the following corollary.

Corollary 3.2 If $\left(\mathrm{X}, \mathcal{C}_{\mathrm{X}}\right)$ is a semi-convex space that satisfies the conditions of the Kernel Theorem, then $\mathcal{C}_{\mathbf{X}}-\operatorname{kernel}(\mathbf{Y})$ is convex, for all $\mathbf{Y} \subseteq \mathbf{X}$.

We call semi-convex spaces that satisfy Condition 3 of the Kernel Theorem near-simple. Simple semi-convex spaces are a special case of near-simple semi-convex spaces when the set $\mathbf{C}_{0}$ of Condition 3 is empty. As an example of a near-simple semi-convex space that is not simple but for which the Kernel Theorem holds consider a simple polygon $\mathbf{X}$ in the plane. Let $\mathbf{C}_{0}$ be some nonempty subset of $\mathbf{X}$ and $\mathcal{C}$ be the subsets of $\mathbf{X}$ that are Euclidean convex. Let $\mathcal{C}_{\mathbf{X}}=\left\{\mathbf{C} \cup \mathbf{C}_{0} \mid \mathbf{C} \in \mathcal{C}\right\}$. We claim that $\left(\mathbf{X}, \mathcal{C}_{\mathbf{X}}\right)$ satisfies the Kernel Theorem. It clearly satisfies Conditions 1 and 3 . To see that it satisfies Condition 2 let $x$ be a point in $\mathbf{X}$ and $\mathbf{C} \in \mathcal{C}$ such that, for each $c \in \mathbf{C} \cup \mathbf{C}_{0}, \mathcal{C}_{\mathbf{X}^{-}} \operatorname{hull}(\{x, c\})=\overline{x c} \cup \mathbf{C}_{0} \subseteq \mathbf{X}$, where $\overline{x c}$ denotes the line segment from $x$ to $c$. Hence, $\mathcal{C}_{\mathbf{X}^{-}}$join $\left(x, \mathbf{C} \cup \mathbf{C}_{0}\right)$ is the Euclidean convex hull of $\{x\} \cup \mathbf{C} \cup \mathbf{C}_{0}$ which belongs to $\mathcal{C}_{\mathbf{X}}$ as claimed.

\subsection{Convexity of Kernels}

The Kernel Theorem gives rise to two natural follow up questions.

1. Can we guarantee the convexity of kernels under weaker conditions than those necessary for the Kernel Theorem? 
2. Is there a relationship between kernels and skulls in general semiconvex spaces?

Let us consider the convexity of kernels first. Algorithms that compute kernels of planar sets often make crucial use of the fact that they are convex. Therefore, it is desirable to have a characterization of those semi-convex spaces for which this is true. Unfortunately, there is a large gap between the sufficient and necessary conditions for the convexity of kernels as stated in the following theorems.

Theorem 3.3 Let $\left(\mathrm{X}, \mathcal{C}_{\mathbf{X}}\right)$ be a complete semi-convex space. If $\left(\mathrm{X}, \mathcal{C}_{\mathbf{X}}\right)$ satisfies the join condition, then $\mathcal{C}_{\mathbf{X}}-\operatorname{kernel}(\mathbf{Y})$ is convex, for all $\mathbf{Y} \subseteq \mathbf{X}$.

Proof: Let $\mathbf{Y} \subseteq \mathbf{X}$ and, without loss of generality, we assume that $\mathcal{C}_{\mathbf{X}}-\operatorname{kernel}(\mathbf{Y}) \neq \emptyset$. Consider $x, y \in \mathcal{C}_{\mathbf{X}}-\operatorname{kernel}(\mathbf{Y})$. Since $x$ and $y$ can see each other, we have $\mathcal{C}_{\mathbf{X}}-\operatorname{hull}(\{x, y\}) \neq \emptyset$ and $\mathcal{C}_{\mathbf{X}}-h u l l(\{x, y\}) \subseteq \mathbf{Y}$. Now take any $z \in \mathbf{Y}$. We want to show that any point $w$ in $\mathcal{C}_{\mathbf{X}}-h u l l(\{x, y\})$ can see $z$. This implies that $w$ belongs to $\mathcal{C}_{\mathbf{X}^{-}} \operatorname{kernel}(\mathbf{Y})$ and, hence, $\mathcal{C}_{\mathbf{X}^{-}} \operatorname{kernel}(\mathbf{Y})$ is visibility convex.

Let $\mathbf{Q}$ be defined as $\mathcal{C}_{\mathbf{X}^{-}} \operatorname{join}\left(x, \mathcal{C}_{\mathbf{X}^{-}} h u l l(\{y, z\})\right)$. We have $\mathbf{Q} \subseteq \mathbf{Y}$ and $\mathbf{Q}$ is nonempty since $\emptyset \neq \mathcal{C}_{\mathbf{X}^{-}} h u l l(\{y, z\}) \subseteq \mathbf{Y}$ and $x$ can see all points in $\mathcal{C}_{\mathbf{X}}-\operatorname{hull}(\{y, z\})$. Now let $w$ be a point in $\mathcal{C}_{\mathbf{X}}-\operatorname{hull}(\{x, y\})$. Since, $\mathbf{Q}=\mathcal{C}_{\mathbf{X}^{-}} \operatorname{hull}(\{x, y, z\})=\mathcal{C}_{\mathbf{X}}-j \operatorname{oin}\left(z, \mathcal{C}_{\mathbf{X}^{-}} h u l l(\{x, y\})\right)$ by the joinproperty, we have $\mathcal{C}_{\mathbf{X}}-h u l l(\{w, z\}) \subseteq \mathbf{Y}$. Further, $\mathcal{C}_{\mathbf{X}}-h u l l(\{w, z\}) \neq \emptyset$ because $\mathcal{C}_{\mathbf{X}^{-}} \operatorname{hull}(\{w, z\}) \subseteq \mathcal{C}_{\mathbf{X}^{-}} \operatorname{hull}(\{x, y, z\}) \neq \emptyset$. But this implies that $w \in \mathcal{C}_{\mathbf{X}}-\operatorname{kernel}(\mathbf{Y})$, since $z$ was arbitrary. Hence, $\mathcal{C}_{\mathbf{X}}-\operatorname{kernel}(\mathbf{Y})$ is visibility convex and by the completeness of $\left(\mathbf{X}, \mathcal{C}_{\mathbf{X}}\right)$ it is convex.

The assumption of a complete space cannot be relaxed as the next theorem shows.

Theorem 3.4 If $\left(\mathrm{X}, \mathcal{C}_{\mathrm{X}}\right)$ is a semi-convex space such that, for all $\mathrm{Y} \subseteq \mathrm{X}$, $\mathcal{C}_{\mathbf{X}}-$ kernel $(\mathbf{Y})$ is convex, then $\left(\mathbf{X}, \mathcal{C}_{\mathbf{X}}\right)$ is complete.

Proof: Let $\mathbf{C} \subseteq \mathbf{X}$ be a visibility convex set. Since we then have $\mathcal{C}_{\mathbf{X}}$ kernel $(\mathbf{C})=\mathbf{C}$, the convexity of kernels immediately implies that $\mathrm{C} \in \mathcal{C}_{\mathbf{X}}$. 


\subsection{An Extension to the Kernel Theorem}

We turn now to the second question: What is the relationship between $\mathcal{C}_{\mathbf{X}}-$ skulls $(\mathbf{Y})$ and $\mathcal{C}_{\mathbf{X}}-$ kernel $(\mathbf{Y})$, for $\mathbf{Y} \subseteq \mathbf{X}$, in an arbitrary semi-convex space? It is obvious that we have to require the existence of skulls in order to be able to make a statement that relates skulls and kernels. Taking this condition into account the next theorem gives the most general connection between skulls and kernels possible.

But before we can state and prove a generalized version of the Kernel Theorem, we need the notion of a skull cover.

Definition 3.1 Given a semi-convex space $\left(\mathrm{X}, \mathcal{C}_{\mathbf{X}}\right)$ and a set $\mathrm{Y} \subseteq \mathrm{X}$ :

1. A subset $\mathcal{S}$ of $\mathcal{C}_{\mathbf{X}}-$ skulls $(\mathbf{Y})$ is a skull cover of $\mathbf{Y}$ if $\bigcup \mathcal{S}=\mathbf{Y}$.

2. A subset $\mathcal{S}$ of $\mathcal{C}_{\mathrm{X}}-$ skulls $(\mathrm{Y})$ is a minimal skull cover of $\mathbf{Y}$ if $\mathcal{S}$ is a skull cover of $\mathbf{Y}$ and, for all $\mathbf{S} \in \mathcal{S}, \mathcal{S} \backslash\{\mathbf{S}\}$ is not a skull cover of $\mathbf{Y}$.

We denote the set of all skull covers of $\mathbf{Y}$ by $\Sigma(\mathbf{Y})$.

The theorem can now be stated as follows.

Theorem 3.5 (The Cover Kernel Theorem) If $\left(\mathrm{X}, \mathcal{C}_{\mathbf{X}}\right)$ is a semiconvex space, then we have, for all $\mathrm{Y} \subseteq \mathrm{X}$, that

$$
\mathcal{C}_{\mathbf{X}}-\operatorname{kernel}(\mathbf{Y})=\bigcup_{\mathcal{S} \in \Sigma(\mathbf{Y})}(\bigcap \mathcal{S})
$$

if, for all convex subsets $\mathbf{C}$ of $\mathbf{Y}$, there is an $\mathbf{S} \in \mathcal{C}_{\mathbf{X}}-$ skulls $(\mathbf{Y})$ with $\mathbf{C} \subseteq \mathbf{S}$.

Proof: Let $\mathbf{Y}$ be a nonempty subset of $\mathbf{X}, K=\mathcal{C}_{\mathbf{X}}-\operatorname{kernel}(\mathbf{Y})$, and $I=$ $\bigcup_{\mathcal{S} \in \Sigma(\mathbf{Y})}(\cap \mathcal{S})$. We first prove that $K \subseteq I$ and then prove that $I \subseteq K$.

$K \subseteq I$. Without loss of generality assume that $K \neq \emptyset$. Let $x \in K$ and define $\mathcal{S}_{x}=\left\{\mathbf{S} \in \mathcal{C}_{\mathbf{X}^{-}}\right.$skulls $\left.(\mathbf{Y}) \mid x \in \mathbf{S}\right\}$. Since $x$ can see all points in $\mathbf{Y}$, we have that, for all $y \in \mathbf{Y}, \emptyset \neq \mathcal{C}_{\mathbf{X}}-\operatorname{hull}(\{x, y\}) \subseteq \mathbf{Y}$; that is, there exists an $\mathbf{S} \in \mathcal{S}_{x}$ such that $\{x, y\} \subseteq \mathcal{C}_{\mathbf{X}}-h u l l(\{x, y\}) \subseteq \mathbf{S} \in \mathcal{S}_{x}$ since the existence of skulls for any convex subset $\mathbf{C}$ of $\mathbf{Y}$ is guaranteed. Hence, $\mathcal{S}_{x}$ is a cover of $\mathbf{Y}$ with $x \in \bigcap \mathcal{S}_{x} \subseteq I$.

$I \subseteq K$. Let $x \in I$; then, there is an $\mathcal{S} \in \Sigma(\mathbf{Y})$ such that $x \in \cap \mathcal{S}$. Let $y$ be an arbitrary point in $\mathbf{Y}$; then, since $\mathcal{S}$ is a cover of $\mathbf{Y}$, there is a skull $\mathbf{S} \in \mathcal{S}$ such that $y \in \mathbf{S}$. Because $x \in \cap \mathcal{S}$, we know that $x \in \mathbf{S}$, so $\{x, y\} \subseteq \mathbf{S} ;$ since $\mathcal{C}_{\mathbf{X}^{-}}$hull $(\{x, y\}) \neq \emptyset$ and $\mathcal{C}_{\mathbf{X}}-h u l l(\{x, y\}) \subseteq \mathbf{S} \subseteq \mathbf{Y}$, we have $x \mathcal{C}_{\mathbf{Y}^{-}}$sees $y$ and $x \in \mathcal{C}_{\mathbf{X}^{-}} \operatorname{kernel}(\mathbf{Y})$. 
Clearly, it is possible to restrict ourselves to minimal skull covers in the above theorem. This gives us a slightly stronger version of the first Kernel Theorem.

Corollary 3.6 If $\left(\mathrm{X}, \mathcal{C}_{\mathbf{X}}\right)$ be a semi-convex space that satisfies the three conditions of the Kernel Theorem, then we have, for all $\mathrm{Y} \subseteq \mathrm{X}$ and all minimal skull covers $\mathcal{S}$ of $\mathbf{Y}$,

$$
\mathcal{C}_{\mathbf{X}}-\operatorname{kernel}(\mathbf{Y})=\bigcap \mathcal{S} .
$$

Proof: If $\mathcal{S}_{0}$ is some minimal cover of $\mathbf{Y}$, then

$$
\begin{aligned}
& \mathcal{C}_{\mathbf{X}^{-}} \operatorname{kernel}(\mathbf{Y})=\bigcap \mathcal{C}_{\mathbf{X}^{-}} \text {skulls }(\mathbf{Y}) \quad \text { by the Kernel Theorem } \\
& \subseteq \bigcap \mathcal{S}_{0} \\
& \subseteq \bigcup_{\mathcal{S} \in \Sigma(\mathbf{Y})}(\cap \mathcal{S}) \\
& =\mathcal{C}_{\mathbf{X}^{-}} \operatorname{kernel}(\mathbf{Y}) \text {. }
\end{aligned}
$$

We conjecture that the converse of the Cover Kernel Theorem does not hold, that is, there are semi-convex spaces for which the kernel of a subset equals the union of the intersections of skull covers, for all subsets but there are convex sets that are not contained in skulls; however, we have not been able to construct an explicit counterexample. The question then remains what other conditions could be imposed to guarantee the existence of skulls.

\section{Semi-convex Space Construction}

This section is concerned with different methods of constructing semi-convex spaces. If the family of convex sets is not given by enumeration which is, of course, only possible if we consider finite semi-convex spaces, then the convexity of a set depends on a specific condition that has to be satisfied. The most commonly used condition is to require the "hull" of any two points to be contained in the considered set. As an example consider the usual convexity in the plane which is based on line segments as the hull of two points.

\subsection{The Hull Function}

So suppose we are given a function hull $: \mathbf{X} \times \mathbf{X} \longrightarrow 2^{\mathbf{X}}$ and specify our family of convex sets $\mathcal{C}_{\mathbf{X}}$ by $\mathcal{C}_{\mathbf{X}}=\{\mathbf{C} \subseteq \mathbf{X} \mid$ for all $x$ and $y \in \mathbf{C}:\{x, y\} \subseteq$ 
$\operatorname{hull}(x, y) \subseteq \mathbf{C}\}$. We show that this is, of course, the definition of a complete semi-convex space.

Theorem 4.1 If hull is a function from $\mathrm{X} \times \mathrm{X}$ into $2^{\mathrm{X}}$, then $\left(\mathrm{X}, \mathcal{C}_{\mathbf{X}}\right)$ according to the previous definition is a complete semi-convex space.

Proof: Note that the empty set satisfies the above definition of convex set. Therefore, we only have to show the closure under intersection. Let $\mathcal{C} \subseteq \mathcal{C}_{\mathbf{X}}$ be a family of convex sets and $x, y \in \bigcap \mathcal{C}$. Since $h u l l(x, y) \subseteq \mathbf{C}$, for all $\mathrm{C} \in \mathcal{C}$, have $\operatorname{hull}(x, y) \subseteq \bigcap \mathcal{C}$. Hence, $\cap \mathcal{C}$ satisfies the above definition and is $\mathcal{C}_{\mathbf{X}}$-convex. $\left(\mathbf{X}, \mathcal{C}_{\mathbf{X}}\right)$ is complete because if $\mathbf{C}$ is a subset of $\mathbf{X}$ such that, for all $x, y \in \mathbf{C}$, we have $\mathcal{C}_{\mathbf{X}}-h u l l(\{x, y\}) \subseteq \mathbf{C}$, then, clearly, hull $(x, y) \subseteq$ $\mathcal{C}_{\mathbf{X}}-h u l l(\{x, y\})$ by the definition of $\mathcal{C}_{\mathbf{X}}-h u l l(\{x, y\})$ and $\mathbf{C}$ is convex.

An interesting question, which we answer immediately, is: When, for all $x, y$, is $\operatorname{hull}(x, y)$ equal to $\mathcal{C}_{\mathbf{X}}-h u l l(\{x, y\})$ ?

Theorem 4.2 Let hull and $\mathcal{C}_{\mathbf{X}}$ be defined as above. Then, for all $x, y \in \mathbf{X}$, $\operatorname{hull}(x, y)=\mathcal{C}_{\mathbf{X}}-h u l l(\{x, y\})$ if and only if, for all $p, q \in \operatorname{hull}(x, y),\{p, q\} \subseteq$ $\operatorname{hull}(p, q) \subseteq \operatorname{hull}(x, y)$ and $\{x, y\} \subseteq \operatorname{hull}(x, y)$, if hull $(x, y) \neq \emptyset$.

Let hull and $\mathcal{C}_{\mathbf{X}}$ be defined as above. Then, for all $x, y \in \mathbf{X}, \operatorname{hull}(x, y)=$ $\mathcal{C}_{\mathbf{X}}-h u l l(\{x, y\})$ if and only if the following two conditions hold:

1. If hull $(x, y) \neq \emptyset$, then $\{x, y\} \subseteq \operatorname{hull}(x, y)$.

2. If hull $(x, y) \neq \emptyset$, then, for all $p, q \in \operatorname{hull}(x, y),\{p, q\} \subseteq \operatorname{hull}(p, q) \subseteq$ $\operatorname{hull}(x, y)$.

Proof: The necessity of the condition follows immediately from the properties of the hull-operator. So we only have to show the sufficiency. Clearly, we have $\operatorname{hull}(x, y) \subseteq \mathcal{C}_{\mathbf{X}}-h u l l(\{x, y\})$ so we only have to show the reverse inclusion.

Note that if $\operatorname{hull}(x, y) \neq \emptyset$, then $\operatorname{hull}(x, y)$ is $\mathcal{C}_{\mathbf{X}^{-}}$convex by definition since, for all $p, q \in \operatorname{hull}(x, y),\{p, q\} \subseteq \operatorname{hull}(p, q) \subseteq \operatorname{hull}(x, y)$. Furthermore, hull $(x, y)$ contains $\{x, y\}$. Hence, the inclusion follows since $\mathcal{C}_{\mathbf{X}^{-}} \operatorname{hull}(\{x, y\})$ is the smallest convex set that contains $\{x, y\}$. If $h u l l(x, y)=\emptyset$, then there is no convex set that contains $x$ and $y$ since we defined a convex set $\mathbf{C}$ to have $\operatorname{hull}\left(x^{\prime}, y^{\prime}\right) \neq \emptyset$, for all $x^{\prime}, y^{\prime} \in \mathbf{C}$.

\subsection{Distance Functions and Metrics in the Plane}

Although the method outlined above of defining semi-convex spaces works satisfactorily in most cases since only the hull of two points has to be specified, it is sometimes desirable to further simplify the definition or to add 
more structure to the hull function if possible. One way to proceed in these cases is to use distance functions. A distance function $d$ is a mapping $d: \mathrm{X} \times \mathrm{X} \longrightarrow \mathbb{R}_{+}$that satisfies the following two properties, for all $x, y \in \mathbf{X}$ :

1. $d(x, y) \geq 0$ and $d(x, y)=0 \Longleftrightarrow x=y$ (positive definiteness)

$$
\text { 2. } d(x, y)=d(y, x) \quad \text { (symmetry) }
$$

We say that a point $z$ lies between the points $x$ and $y$ with respect to $d$ if $d(x, z)+d(z, y)=d(x, y)$. A $d$-straight path $\mathcal{P}$ from $p$ to $q$ is a shortest path in $\mathbf{X}$ from $p$ to $q$ that consists only of points that lie between $p$ and $q$.

Definition 4.1 Let $\mathrm{X}$ be some set, $d$ a distance function, and $x, y$ be two points in $\mathbf{X}$. We define the $\mathcal{C}_{d}$-hull of $x$ and $y$ as $\bigcup\{\mathcal{P} \mid \mathcal{P}$ is a d-straight path connecting $x$ and $y\}$ and denote it by $\mathcal{C}_{d}-h u l l(\{x, y\})$.

We denote the semi-convex space induced by $d$ by $\left(\mathrm{X}, \mathcal{C}_{d}\right)$. It is not obvious that the above definition conforms with our concept of convex hull and it is not too difficult to find counterexamples of distance functions for which $\mathcal{C}_{d^{-}}$hull is not a hull function.

A very interesting special case of distance functions are metrics, which have received a lot of attention in both computer science and mathematics. A metric $d$ satisfies the additional property:

3. $d(x, z) \leq d(x, y)+d(y, z)$, for all $x, y, z \in \mathbf{X}$. (triangle inequality)

In the following we will show that the semi-convex spaces which are induced by metrics that have certain additional properties satisfy the joincondition. Since the semi-convex spaces induced by distance functions are always simple and complete, this implies that these semi-convex spaces satisfy the Kernel Theorem. From now on throughout the rest of this paper we will assume that $\mathrm{X}$ is a simple domain. A simple domain is a subset of the plane that is homeomorphic to the closure of the unit ball; that is, a subset that is bounded by a simple closed curve.

So let $\mathrm{X}$ be a simple domain and $d$ metric in it. In order to show that $d$ satisfies the join property several conditions have to be met. We say $d$ is straight if $d$ satisfies the condition that there is at least one $d$-straight path in $\mathbb{E}^{2}$ between any two points in $\mathbf{X}$.

The second condition we impose requires that we can prolong $d$-straight paths sufficiently. We say $d$ is extensible if $d$ satisfies the following two conditions. 
(i) For any $d$-straight path $\mathcal{P}$, there is a $d$-straight extension of $\mathcal{P}$ that partitions $\mathbf{X}$ into at least two components and

(ii) all points contained in a $d$-triangle - that is a Jordan curve which consists of three $d$-straight paths - can be connected to the corner points of the $d$-triangle by a $d$-straight path in $\mathrm{X}$.

Finally, we need a third property to ensure that $d$ satisfies the join condition.

If $p, q, r$, and $s$ are four points in $\mathbf{X}$ such that $q$ is in $\mathcal{C}_{d^{-}} \operatorname{hull}(\{p, r\})$ and $r \in \mathcal{C}_{d^{-}} \operatorname{hull}(\{q, s\})$, then $\mathcal{C}_{d^{-}} \operatorname{hull}(\{p, r\})$ is contained in $\mathcal{C}_{d^{-}}$join $\left(q, \mathcal{C}_{d^{-}} h u l l(\{p, s\})\right)$.

A metric that satisfies the above condition is called stiff. Note that any metric that induces a semi-convex space which satisfies the join condition is necessarily stiff since, if $p, q, r$, and $s$ are four points in $\mathrm{X}$ such that $q$ is in $\mathcal{C}_{d^{-}} \operatorname{hull}(\{p, r\})$ and $r \in \mathcal{C}_{d^{-}}$hull $(\{q, s\})$, then $\mathcal{C}_{d^{-}}$join $\left(q, \mathcal{C}_{d^{-}}\right.$hull $\left.(\{p, s\})\right)$ is $d_{-}$ convex and $p$ and $r$ are contained in $\mathcal{C}_{d^{-}}$join $\left(q, \mathcal{C}_{d^{-}} h u l l(\{p, s\})\right)$; this implies that $\mathcal{C}_{d^{-}} h u l l(\{p, r\}) \subseteq \mathcal{C}_{d^{-}}$join $\left(q, \mathcal{C}_{d^{-}}\right.$hull $\left.(\{p, s\})\right)$.

We are now able to state the following theorem about simple domains.

Theorem 4.3 If $\mathrm{X}$ is a simple domain and $d$ is a straight, extensible, and stiff metric in $\mathbf{X}$, then $\left(\mathrm{X}, \mathcal{C}_{d}\right)$ satisfies the join-property.

Since there are a several cases to consider to show the preceding theorem, we start with a number of definitions, lemmas, and observations.

We will be mostly dealing with $d$-straight paths in the plane. Given two curves $\mathcal{P}$ and $\mathcal{Q}$ such that the end point of $\mathcal{P}$ equals the start point of $\mathcal{Q}$ we denote the concatenation of $\mathcal{P}$ and $\mathcal{Q}$ by $\mathcal{P} * \mathcal{Q}$. By the famous Jordan Curve Theorem [Rin 75, 40.1] a simple closed curve $\mathcal{C}$ partitions the plane into two parts, one bounded called the interior of $\mathcal{C}$ and denoted by int $(\mathcal{C})$, and the other unbounded called the exterior of $\mathcal{C}$ and denoted by $\operatorname{ext}(\mathcal{C})$.

Given two points in $\mathrm{X}$, we denote by $\mathcal{P}_{p q}$ a $d$-straight path from $p$ to $q$. We assume that $\mathcal{P}_{p q}$ is endowed with an injective parametrization that has $p$ as start point and $q$ as end point. So, if we talk of points on $\mathcal{P}_{p q}$ being before or after others, this is meant according to the order induced by the parametrization. If $r$ and $s$ are two points on $\mathcal{P}_{p q}$, we denote the part of $\mathcal{P}_{p q}$ from $r$ to $s$ by $\left.\mathcal{P}_{p q}\right|_{r s}$. We denote the inverse path of $\mathcal{P}_{p q}$ from $q$ to $\frac{p}{\mathcal{P}}$ by $\mathcal{P}_{p q}^{-1}$. Furthermore, an extension of a $d$-straight path $\mathcal{P}$ is denoted by $\overline{\mathcal{P}}$ and $\lambda_{d}(\mathcal{P})$ denotes its length. 


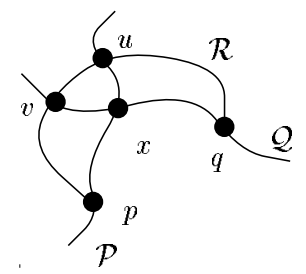

Figure 1: $\left.\left.\mathcal{P}\right|_{p x} * \mathcal{Q}\right|_{x q}$ is a $d$-straight path from $p$ to $q$.

Recall that $d$-straight paths are shortest paths in $\mathbf{X}$ [Rin61, p. 147]. If there exists a $d$-straight path from $p$ to $q$, then any other shortest path from $p$ to $q$ is also $d$-straight. If $r$ and $s$ are two points on $\mathcal{P}_{p q}$, then any shortest path $\mathcal{P}_{r s}$ from $r$ to $s$ is also $d$-straight and so is $\left.\left.\mathcal{P}_{p q}\right|_{p r} * \mathcal{P}_{r s} * \mathcal{P}_{p q}\right|_{s q}$ if $r$ occurs before $s$ on $\mathcal{P}_{p q}$ [Rin61, p. 147].

Since we often deal with choosing a first or last intersection point of two curves, the following observation is useful.

Observation 4.4 Let $\mathcal{P}$ and $\mathcal{Q}$ be two d-straight paths that start in the same point. If $s$ is the first resp. last intersection point of $\mathcal{P}$ and $\mathcal{Q}$ on $\mathcal{P}$, then $s$ is also the first resp. last intersection point of $\mathcal{P}$ and $\mathcal{Q}$ on $\mathcal{Q}$.

A simple and useful observation concerning $d$-straight paths is given in the next lemma.

Lemma 4.5 Let $\mathcal{P}$ and $\mathcal{Q}$ be two d-straight paths that intersect in a point $x$. Furthermore, let $p$ be a point on $\mathcal{P}$ before $x$ and $q$ a point on $\mathcal{Q}$ before $x$. If there is a d-straight path $\mathcal{R}$ from $p$ to $q$ that intersects $\mathcal{P}$ and $\mathcal{Q}$ after $x$, then $\left.\left.\mathcal{P}\right|_{p x} * \mathcal{Q}\right|_{x q}$ is also a d-straight path from $p$ to $q$.

Proof: Let the intersection point of $\mathcal{P}$ with $\mathcal{R}$ be $u$ and the intersection point of $\mathcal{Q}$ with $\mathcal{R}$ be $v$. Note that if $u$ occurs before $v$ on $\mathcal{R}$, then $\lambda_{d}\left(\left.\mathcal{R}\right|_{p u}\right)=$ $\lambda_{d}\left(\left.\mathcal{P}\right|_{p u}\right)=\lambda_{d}\left(\left.\mathcal{P}\right|_{p x}\right)+\lambda_{d}\left(\left.\mathcal{P}\right|_{x u}\right)$ since both $\mathcal{R}$ and $\mathcal{P}$ are $d$-straight paths. Similarly, we obtain $\lambda_{d}\left(\left.\mathcal{R}\right|_{v q}\right) \geq \lambda_{d}\left(\left.\mathcal{Q}\right|_{x q}\right)$ and

$$
\lambda_{d}(\mathcal{R}) \geq \lambda_{d}\left(\left.\mathcal{R}\right|_{p u}\right)+\lambda_{d}\left(\left.\mathcal{R}\right|_{v q}\right) \geq \lambda_{d}\left(\left.\mathcal{P}\right|_{p x}\right)+\lambda_{d}\left(\left.\mathcal{Q}\right|_{x q}\right) .
$$

Therefore, $\left.\left.\mathcal{P}\right|_{p x} * \mathcal{Q}\right|_{x q}$ is a $d$-straight path from $p$ to $q$.

So assume that $u$ occurs after $v$ on $\mathcal{R}$ (see Figure 1 ). Hence, $\lambda_{d}(\mathcal{R})=$ 
$\lambda_{d}\left(\left.\mathcal{R}\right|_{p v}\right)+\lambda_{d}\left(\left.\mathcal{R}\right|_{v u}\right)+\lambda_{d}\left(\left.\mathcal{R}\right|_{u q}\right)$. With $\lambda_{d}\left(\left.\mathcal{R}\right|_{p u}\right)=\lambda_{d}\left(\left.\mathcal{P}\right|_{p u}\right)$ and $\lambda_{d}\left(\left.\mathcal{R}\right|_{v q}\right)=$ $\lambda_{d}\left(\left.\mathcal{Q}\right|_{v q}\right)$ we obtain

$$
\begin{aligned}
\lambda_{d}(\mathcal{R}) & =\lambda_{d}\left(\left.\mathcal{R}\right|_{p v}\right)+\lambda_{d}\left(\left.\mathcal{R}\right|_{v u}\right)+\lambda_{d}\left(\left.\mathcal{R}\right|_{u q}\right) \\
& =\lambda_{d}\left(\left.\mathcal{P}\right|_{p u}\right)+\lambda_{d}\left(\left.\mathcal{Q}\right|_{v q}\right)-\lambda_{d}\left(\left.\mathcal{R}\right|_{v u}\right) \\
& =\lambda_{d}\left(\left.\mathcal{P}\right|_{p x}\right)+\lambda_{d}\left(\left.\mathcal{Q}\right|_{x q}\right)+\lambda_{d}\left(\left.\mathcal{P}\right|_{x u}\right)+\lambda_{d}\left(\left.\mathcal{Q}\right|_{v x}\right)-\lambda_{d}\left(\left.\mathcal{R}\right|_{v u}\right) \\
& \geq \lambda_{d}\left(\left.\mathcal{P}\right|_{p x}\right)+\lambda_{d}\left(\left.\mathcal{Q}\right|_{x q}\right)
\end{aligned}
$$

where the last inequality follows from the triangle inequality. Hence, $\left.\mathcal{P}\right|_{p x} *$ $\left.\mathcal{Q}\right|_{x q}$ is a $d$-straight path from $p$ to $q$ as claimed.

The $\mathcal{C}_{d^{-}}$join depends on the properties of the $\mathcal{C}_{d}$-hull. Hence, we will first show that the $\mathcal{C}_{d^{-}}$hull satisfies the definition of a convex hull.

Lemma 4.6 Let $d$ be a straight, extensible, and stiff metric and $x, y$ be two points in $\mathrm{X}$ with nonempty $\mathcal{C}_{d}$-hull. If $p, q \in \mathcal{C}_{d}$-hull $(\{x, y\})$, then $\mathcal{C}_{d}-h u l l(\{p, q\}) \subseteq \mathcal{C}_{d}-h u l l(\{x, y\})$.

Proof: Let $\mathcal{C}_{p}, \mathcal{C}_{q}$ be two $d$-straight curves from $x$ to $y$ with $p \in \mathcal{C}_{p}$ and $q \in \mathcal{C}_{q}$ and let $\mathcal{P}$ be a $d$-straight path from $p$ to $q$. We have to show that $\mathcal{P}$ belongs to $\mathcal{C}_{d}$ hull $(\{x, y\})$. Let $p^{\prime}$ be the last intersection point of $\mathcal{C}_{p}$ with $\mathcal{P}\left(\right.$ on $\mathcal{P}$ ) and $q^{\prime}$ the first intersection point of $\mathcal{C}_{q}$ with $\mathcal{P}($ on $\mathcal{P})$. Note that the parts of $\mathcal{P}$ from $p$ to $p^{\prime}$ and from $q^{\prime}$ to $q$ are parts of a $d$-straight path from $x$ to $y$. Hence, we assume w.l.o.g. that $p^{\prime}=p$ and $q^{\prime}=q$.

Let $x^{\prime}$ be the last intersection point of $\mathcal{C}_{p}$ and $\mathcal{C}_{q}$ before $p$ and $q$ and $y^{\prime}$ the first intersection point of $\mathcal{C}_{p}$ and $\mathcal{C}_{q}$ after $p$ and $q$. If $\mathcal{C}_{p}$ and $\mathcal{C}_{q}$ intersect in a point $u$ between $p$ and $q$, then

$$
\begin{aligned}
\lambda_{d}\left(\mathcal{C}_{p}\right) & =\lambda_{d}\left(\left.\mathcal{C}_{p}\right|_{x u}\right)+\lambda_{d}\left(\left.\mathcal{C}_{q}\right|_{u y}\right) \\
& =\lambda_{d}\left(\left.\mathcal{C}_{p}\right|_{x p}\right)+\lambda_{d}\left(\left.\mathcal{C}_{p}\right|_{p u}\right)+\lambda_{d}\left(\left.\mathcal{C}_{q}\right|_{u q}\right)+\lambda_{d}\left(\left.\mathcal{C}_{q}\right|_{q y}\right) \\
& \geq \lambda_{d}\left(\left.\mathcal{C}_{p}\right|_{x p}\right)+\lambda_{d}(\mathcal{P})+\lambda_{d}\left(\left.\mathcal{C}_{q}\right|_{q y}\right)
\end{aligned}
$$

and $\mathcal{P}$ is part of a $d$-straight path from $x$ to $y$.

If the curve $\left.\left.\mathcal{C}_{p}\right|_{x^{\prime} y^{\prime}} * \mathcal{C}_{q}\right|_{y^{\prime} x^{\prime}}$ is a simple closed curve $\mathcal{C}$ and $\mathcal{P}$ does not intersect the interior of $\mathcal{C}$, then a $d$-straight path from $p$ to $q$ that does not intersect $\mathcal{C}_{p}$ either intersects both extensions $\overline{\mathcal{C}}_{p}$ and $\overline{\mathcal{C}}_{q}$ before $x$ or after $y$. By Lemma 4.5 and the stiffness of $d$ this implies that $\mathcal{C}_{p} * \mathcal{C}_{q}^{-1}$ is a $d$-straight path which is a contradiction. Hence, if $\left.\left.\mathcal{C}_{p}\right|_{x^{\prime} y^{\prime}} * \mathcal{C}_{q}\right|_{y^{\prime} x^{\prime}}$ is a simple closed curve $\mathcal{C}$, then $\mathcal{P}$ is contained in the interior of $\mathcal{C}$.

So let $r$ be a point on $\mathcal{P}$ and $\mathcal{R}$ a $d$-straight path from $x$ to $r$. The extension $\overline{\mathcal{R}}$ of $\mathcal{R}$ intersects $\mathcal{C}$ and, hence, either $\mathcal{C}_{p}$ or $\mathcal{C}_{q}$. Therefore, $r$ is on a $d$-straight path from $x$ to $y$ as claimed. 
We now turn to the proof of Theorem 4.3. Since the considered semiconvex space is complete, it suffices to prove the join-property for three points.

Let $x, y$, and $z$ be three points in $\mathbf{X}$ and let $\mathbf{Q}$ be defined as $\mathcal{C}_{d^{-}}$join $\left(x, \mathcal{C}_{d^{-}}\right.$hull $\left.(\{y, z\})\right)$. Consider two points $p, q \in \mathbf{Q}$ and a $d$-straight path $\mathcal{P}_{p q} \subseteq \mathcal{C}_{d^{-}}$hull $(\{p, q\})$. We have to show that $\mathcal{P}$ belongs to $\mathbf{Q}$. The convexity of $\mathbf{Q}$ then follows by the completeness of $\left(\mathbf{X}, \mathcal{C}_{d}\right)$.

Let $y_{p}$ and $z_{q}$ be two points in $\mathcal{C}_{d^{-}} h u l l(\{y, z\})$ such that $p \in$ $\mathcal{C}_{d^{-}} \operatorname{hull}\left(\left\{x, y_{p}\right\}\right)$ and $q \in \mathcal{C}_{d^{-}} \operatorname{hull}\left(\left\{x, z_{q}\right\}\right)$. By Lemma 4.6 it suffices to prove that $\mathcal{C}_{d^{-}}$hull $(\{p, q\}) \subseteq \mathcal{C}_{d^{-}}$join $\left(x, \mathcal{C}_{d^{-}}\right.$hull $\left.\left(\left\{y_{p}, z_{q}\right\}\right)\right)$. So w.l.o.g. suppose that $y=y_{q}$ and $z=z_{q}$. Let $\mathcal{P}_{p q}$ be some $d$-straight path in $\mathcal{C}_{d^{-}} \operatorname{hull}(\{p, q\})$. Further, let $\mathcal{P}_{x y}, \mathcal{P}_{x z}$, and $\mathcal{P}_{y z}$ be $d$-straight paths from $x$ to $y, x$ to $z$, and $y$ to $z$. As in the proof of Lemma 4.6 let $p^{\prime}$ be the last intersection point of $\mathcal{P}_{p q}$ with $\mathcal{P}_{x y}$ (on $\mathcal{P}_{p q}$ ) and $q^{\prime}$ the first intersection point of $\mathcal{P}_{p q}$ with $\mathcal{P}_{x z}$. Since the part of $\mathcal{P}_{p q}$ from $p$ to $p^{\prime}$ belongs to $\mathcal{C}_{d}-h u l l(\{x, y\})$ and the part of $\mathcal{P}_{p q}$ from $q^{\prime}$ to $q$ belongs to $\mathcal{C}_{d^{-}} \operatorname{hull}(\{x, z\})$ by Lemma 4.6 , we assume in the following that $p^{\prime}=p$ and $q^{\prime}=q$ and that $\mathcal{P}_{p q}$ does intersect neither $\mathcal{P}_{x y}$ nor $\mathcal{P}_{x z}$.

Let $x^{\prime}$ be the last intersection point of $\mathcal{P}_{x y}$ with $\mathcal{P}_{x z}$. Similarly, let $y^{\prime}$ be the last intersection point of $\mathcal{P}_{x y}^{-1}$ with $\mathcal{P}_{y z}$ and $z^{\prime}$ be the last intersection point of $\mathcal{P}_{x z}^{-1}$ with $\mathcal{P}_{y z}^{-1}$.

Lemma 4.7 If $p$ belongs to the part of $\mathcal{P}_{x y}$ before $x^{\prime}$ or $q$ belongs to the part of $\mathcal{P}_{x z}$ before $x^{\prime}$, then $\mathcal{P}_{p q} \subseteq \mathbf{Q}$.

Proof: First note that if $p$ belongs to the part of $\mathcal{P}_{x y}$ before $x^{\prime}$ and $q$ belongs to the part of $\mathcal{P}_{x z}$ before $x^{\prime}$, then $\mathcal{P}_{p q} \subseteq \mathcal{C}_{\mathbf{X}^{-}} \operatorname{hull}\left(\left\{x, x^{\prime}\right\}\right) \subseteq \mathcal{C}_{\mathbf{X}^{-}} h u l l(\{x, y\})$ by Lemma 4.6 .

Hence, w.l.o.g. we can assume that $\left.p \in \mathcal{P}_{x y}\right|_{x x^{\prime}}$ and that $\left.q \in \mathcal{P}_{x z}\right|_{x^{\prime} z}$. Consider the path $\mathcal{Q}=\left.\left.\mathcal{P}_{x y}\right|_{x p} * \mathcal{P}_{p q} * \mathcal{P}_{x z}\right|_{q z}$. Clearly,

$$
\begin{aligned}
\lambda_{d}\left(\mathcal{P}_{x z}\right) & =\lambda_{d}\left(\left.\mathcal{P}_{x y}\right|_{x x^{\prime}}\right)+\lambda_{d}\left(\left.\mathcal{P}_{x z}\right|_{x^{\prime} z}\right) \\
& =\lambda_{d}\left(\left.\mathcal{P}_{x y}\right|_{x p}\right)+\lambda_{d}\left(\left.\mathcal{P}_{x y}\right|_{p x^{\prime}}\right)+\lambda_{d}\left(\left.\mathcal{P}_{x z}\right|_{x^{\prime} q}\right)+\lambda_{d}\left(\left.\mathcal{P}_{x z}\right|_{q z}\right) \\
& \geq \lambda_{d}(\mathcal{Q})
\end{aligned}
$$

and, therefore, $\mathcal{Q}$ is a $d$-straight path from $x$ to $z$; hence, $\mathcal{P}_{p q} \subseteq$ $\mathcal{C}_{\mathbf{X}}-\operatorname{hull}(\{x, z\}) \subseteq \mathbf{Q}$ as claimed.

Let $\mathcal{C}$ be the simple closed curve formed by the concatenation of the part of $\mathcal{P}_{x y}$ from $x^{\prime}$ to $y^{\prime}$ with the part of $\mathcal{P}_{y z}$ from $y^{\prime}$ to $z^{\prime}$ and the part of $\mathcal{P}_{x z}$ from $z^{\prime}$ to $x^{\prime}$. 


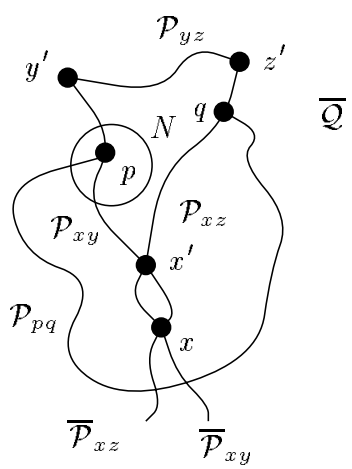

(a)

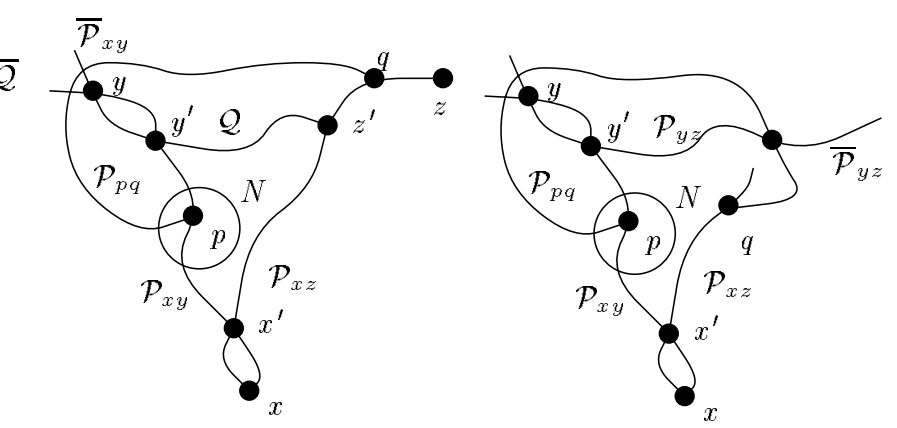

(b)

(c)

Figure 2: The different cases when $\mathcal{P}_{p q}$ intersects the exterior of $\mathcal{C}$ in $N$.

Lemma 4.8 All points in the interior of $\mathcal{C}$ belong to $\mathbf{Q}$.

Proof: Let $u$ be a point in the interior of $\mathcal{C}$ and $\mathcal{P}$ a $d$-straight path from $x$ to $u$. The extension $\overline{\mathcal{P}}$ of $\mathcal{P}$ intersects $\mathcal{C}$. If $\overline{\mathcal{P}}$ intersects $\mathcal{P}_{x y}$ (or $\mathcal{P}_{x z}$ ) in a point $v$, then $\left.\left.\overline{\mathcal{P}}\right|_{x v} * \mathcal{P}_{x y}\right|_{v y}$ is a $d$-straight path and, $u \in \mathcal{C}_{\mathbf{X}}-h u l l(\{x, y\})$. If $\overline{\mathcal{P}}$ intersects $\mathcal{P}_{y z}$, then $u \in \mathcal{C}_{\mathbf{X}^{-}}$join $\left(x, \mathcal{C}_{\mathbf{X}^{-}}\right.$hull $\left.(\{y, z\})\right)=\mathbf{Q}$ as claimed.

Lemma 4.9 If $p$ belongs to the part of $\mathcal{P}_{x y}$ between $x^{\prime}$ and $y^{\prime}$ or $q$ belongs to the part of $\mathcal{P}_{x z}$ between $x^{\prime}$ and $z^{\prime}$, then $\mathcal{P}_{p q}$ belongs to $\mathbf{Q}$.

Proof: Without loss of generality assume that $p$ belongs to the part of $\mathcal{P}_{x y}$ between $x^{\prime}$ and $y^{\prime}$. Consider a neighbourhood $N$ of $p$ that intersects $\mathcal{C}$ only in $\mathcal{P}_{x y} . \mathcal{P}_{p q}$ intersects either the interior or the exterior of $\mathcal{C}$ in $N$.

Case $1 \mathcal{P}_{p q}$ intersects the exterior of $\mathcal{C}$ in $N$.

In this case $\mathcal{P}_{p q}$ either intersects $\overline{\mathcal{P}}_{x y}$ and $\overline{\mathcal{P}}_{x z}$ before $x$ or $\overline{\mathcal{P}}_{x y}$ and $\overline{\mathcal{P}}_{z y}$ after $y$. In the first case $\left.\left.\mathcal{P}_{x y}\right|_{p x} * \mathcal{P}_{x z}\right|_{x q}$ is a $d$-straight path by Lemma 4.5 (see Figure 2a). The stiffness of $d$ implies that $x \in \mathcal{C}_{\mathbf{X}^{-}} \operatorname{hull}(\{y, z\})=\mathbf{Q}$ and the claim follows by Lemma 4.6.

Now consider the the second case. If $q$ belongs to the part of $\mathcal{P}_{x z}$ after $z^{\prime}$, then $\lambda_{d}\left(\mathcal{P}_{y z}\right)=\lambda_{d}\left(\left.\mathcal{P}_{y z}\right|_{y z^{\prime}}\right)+\lambda_{d}\left(\left.\mathcal{P}_{x z}\right|_{z^{\prime} z}\right)$ and $q$ belongs to the $d$-straight path $\mathcal{Q}=\left.\left.\mathcal{P}_{y z}\right|_{y z^{\prime}} * \mathcal{P}_{x z}\right|_{z^{\prime} z}$ from $y$ to $z$. Since $\mathcal{P}_{p q}$ intersects an extension of $\mathcal{P}_{y z}$ before $y$, it also intersects an extension $\overline{\mathcal{Q}}$ of $\mathcal{Q}$ before $y$. Hence, $\mathcal{P}_{p q}$ intersects the extensions $\overline{\mathcal{Q}}$ and $\overline{\mathcal{P}}_{x y}$; Lemma 4.5 then implies that $\left.\left.\mathcal{P}_{x y}\right|_{p y} * \mathcal{Q}\right|_{y q}$ is a 


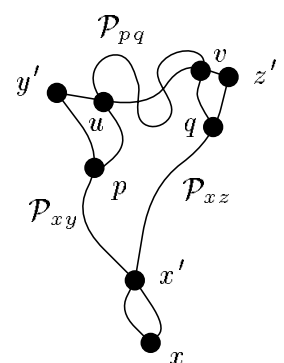

(a)

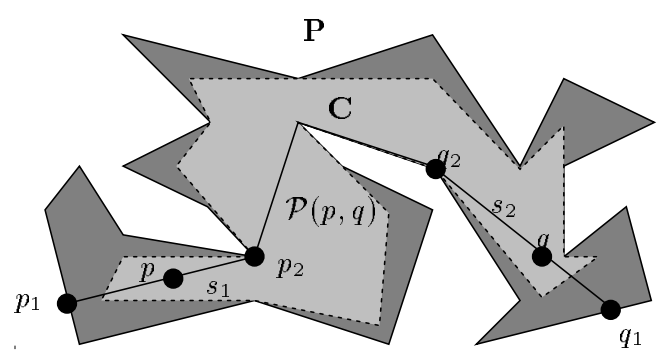

Figure 3: (a) When $\mathcal{P}_{p q}$ intersects the interior of $\mathcal{C}$ in $N$ and $q$ belongs to $\left.\mathcal{P}_{x z}\right|_{x z^{\prime}} \cdot$ (b) An example of a $g$-convex set.

$d$-straight path (see Figure 2b). Again the stiffness of $d$ yields that $y \in$ $\mathcal{C}_{\mathbf{X}}-h u l l(\{x, z\})=\mathbf{Q}$ and, as before, the claim follows from Lemma 4.6.

Finally, if $q$ belongs to the part of $\mathcal{P}_{x z}$ between $x^{\prime}$ and $z^{\prime}$ and $\mathcal{P}_{p q}$ does not intersect $\overline{\mathcal{P}}_{x y}$ and $\overline{\mathcal{P}}_{x z}$ before $x$ (see Figure $2 \mathrm{c}$ ), then $\mathcal{P}_{p q}$ intersects $\overline{\mathcal{P}}_{z y}$ before $y$ and we again obtain a $d$-straight path from $x$ to $z$ via $y$ by Lemma 4.5.

Case $2 \mathcal{P}_{p q}$ intersects the interior of $\mathcal{C}$ in $N$.

If $\mathcal{P}_{p q}$ does not intersect $\mathcal{C}$ again, then $\mathcal{P}_{p q}$ is contained in the interior of $\mathcal{C}$ and belongs to $\mathbf{Q}$ by Lemma 4.8. If $\mathcal{P}_{p q}$ intersects $\mathcal{C}$ in a point $u$, then $u$ belongs to $\mathcal{P}_{y z}$ since $\mathcal{P}_{p q}$ does intersect neither $\mathcal{P}_{x y}$ nor $\mathcal{P}_{x z}$. Without loss of generality let $u$ be the first intersection point of $\mathcal{P}_{p q}$ with $\mathcal{P}_{y z}$. Then, the part of $\mathcal{P}_{p q}$ from $p$ to $u$ is contained in the interior of $\mathcal{C}$ and belongs to $\mathbf{Q}$ again by Lemma 4.8. If $q$ occurs after $z^{\prime}$ on $\mathcal{P}_{x z}$, then $q$ belongs to $\mathcal{C}_{\mathbf{X}}-h u l l(\{y, z\})$ and so does $u$. Hence, the part of $\mathcal{P}_{p q}$ from $u$ to $q$ is also a subset of $\mathcal{C}_{\mathbf{X}^{-}}$hull $(\{y, z\}) \subseteq \mathbf{Q}$. On the other hand, if $q$ is before $z^{\prime}$ on $\mathcal{P}_{x z}$, then let $N^{\prime}$ be a neighbourhood of $q$ that intersects $\mathcal{C}$ only in $\mathcal{P}_{x z}$. If $\mathcal{P}_{p q}$ intersects the exterior of $\mathcal{C}$ in $N^{\prime}$, then we can argue as in Case 1 and $\mathcal{P}_{p q}$ belongs to $\mathbf{Q}$. If $\mathcal{P}_{p q}$ intersects the interior of $\mathcal{C}$ in $N^{\prime}$, then there is a first intersection point $v$ of $\mathcal{P}_{p q}^{-1}$ with $\mathcal{P}_{y z}$ (as seen from $q$ ); for illustration refer to Figure 3. The part of $\mathcal{P}_{p q}$ from $u$ to $v$ belongs to $\mathcal{C}_{\mathbf{X}}-h u l l(\{y, z\})$ while the parts of $\mathcal{P}_{p q}$ from $p$ to $u$ and from $v$ to $q$ are contained in the interior of $\mathcal{C}$ and, therefore, belong to $\mathbf{Q}$ by Lemma 4.8 . 
Lemma 4.10 If $p$ and $q$ belong to the part of $\mathcal{P}_{x y}$ after $y^{\prime}$ and the part of $\mathcal{P}_{x z}$ after $z^{\prime}$, respectively, then $\mathcal{P}_{p q} \subseteq \mathbf{Q}$.

Proof: Clearly, $\left.\left.\mathcal{P}_{x y}\right|_{y p} * \mathcal{P}_{p q} * \mathcal{P}_{x z}\right|_{q z}$ is a $d$-straight path from $y$ to $z$ and, therefore, $\mathcal{P}_{p q} \subseteq \mathcal{C}_{\mathbf{X}}-h u l l(\{y, z\})$. This implies the claim.

Since this enumerates all possible locations of $p$ and $q$ with respect to $x^{\prime}$, $y^{\prime}$, and $z^{\prime}$ we have completed the proof of Theorem 4.3.

\section{$5 \quad$ Examples}

In this section we apply the theory we have developed to two examples.

\subsection{Geodesic Convexity}

Geodesic convexity provides an example of a semi-convex space that has highly non-linear characteristics but still satisfies all the properties we considered in Section 2.

Let $\mathbf{P}$ be a simple polygon in the plane and for any two points $p$ and $q$ in $\mathbf{P}$ let $\mathcal{P}(p, q)$ denote the shortest path lying wholly in $\mathbf{P}$ that connects $p$ and $q$. (We use the standard $L_{2}$-metric to define the distance between two points.) Note that since $\mathbf{P}$ is simple, there is a unique shortest path that connects $p$ and $q$. We say a subset $\mathbf{C}$ of $\mathbf{P}$ is geodesically convex (or g-convex for short) if we have that, for every two points $p$ and $q$ in $\mathbf{C}, \mathcal{P}(p, q)$ belongs to $\mathbf{C}$. Figure $3(\mathrm{~b})$ gives an example. Letting $\mathcal{C}_{g}$ be the set of all $g$-convex sets in $\mathbf{P}\left(\mathbf{P}, \mathcal{C}_{g}\right)$ is a simple and complete semi-convex space.

Furthermore, $\left(\mathbf{P}, \mathcal{C}_{g}\right)$ satisfies the join condition. To show this we need the following lemma.

Lemma 5.1 If $p, q, r$, and $s$ are four points in $\mathbf{P}$ such that $q$ is on $\mathcal{P}(p, r)$ and $r$ is on $\mathcal{P}(q, s)$, then $\mathcal{P}(p, s)$ is the concatenation of $\mathcal{P}(p, r)$ and the part of $\mathcal{P}(q, s)$ from $r$ to $s$.

Proof: The proof is by contradiction. Let $\mathcal{P}$ be the curve consisting of the concatenation of $\mathcal{P}(p, r)$ with the part of $\mathcal{P}(q, s)$ from $r$ to $s$ and assume that $\mathcal{P}$ and $\mathcal{P}(p, s)$ differ. Let $p_{1}$ be the last point on $\mathcal{P}$ such that the part of $\mathcal{P}$ from $p$ to $p_{1}$ equals the part of $\mathcal{P}(p, s)$ from $p$ to $p_{1}$ and let $q_{1}$ be the first intersection point of $\mathcal{P}$ and $\mathcal{P}(p, s)$ after $p_{1}$ on $\mathcal{P}$. For illustration refer to Figure 4(a). If we define $\mathcal{C}$ as the curve consisting of the path of $\mathcal{P}$ from $p_{1}$ to $q_{1}$ concatenated with the part of $\mathcal{P}(p, s)$ from $q_{1}$ to $p_{1}, \mathcal{C}$ is a simple closed curve and, hence, $\operatorname{dom}(\mathcal{C})$ is a simple polygon. The interior angles 


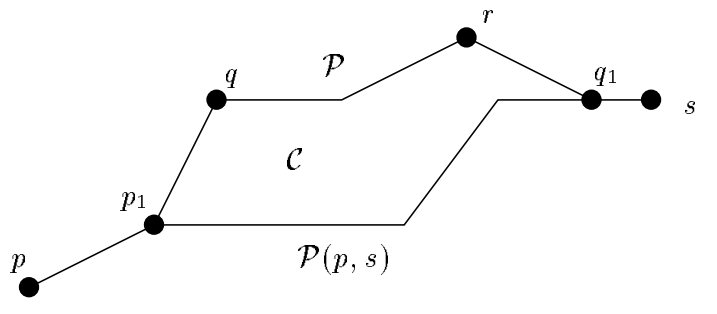

(a)

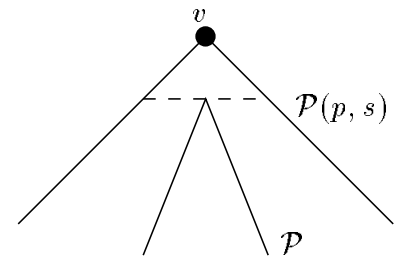

(b)

Figure 4: A convex vertex is shortcut.

at the vertices of $\mathcal{C}$ are reflex except at $p_{1}$ and $q_{1}$ since otherwise we can shorten one of $\mathcal{P}$ or $\mathcal{P}(p, s)$ by "shortcutting" a convex vertex as shown in Figure $4(\mathrm{~b})$. But if $\mathcal{C}$ consists of $n$ vertices, its sum of interior angles is $(n-2) \cdot 180^{\circ}$ which contradicts the fact that we have at least $n-2$ reflex vertices. Hence, $\mathcal{P}$ and $\mathcal{P}(p, s)$ cannot differ.

We now turn to proving that $\left(\mathbf{P}, \mathcal{C}_{g}\right)$ satisfies the join-condition. To this end we, of course, employ Theorem 4.3. So we have to show that the inner metric $d_{\mathbf{P}}$ of $\mathbf{P}$ (that is, the induced metric if we measure the distance between two points $p$ and $q$ according to the length of $\mathcal{P}(p, q))$ is straight, extensible, and stiff.

Clearly, $d_{\mathbf{P}}$ is straight since we measure the length of a path with the Euclidean metric. Furthermore, Lemma 5.1 shows that, for any four points $p, q, r$, and $s$ in $\mathbf{P}$ with $q \in \mathcal{C}_{d_{\mathbf{P}}}-h u l l(\{p, r\})$ and $r \in$ $\mathcal{C}_{d_{\mathbf{P}}}-\operatorname{hull}(\{q, s\})$, we have that $\mathcal{C}_{d_{\mathbf{P}}}-\operatorname{hull}(\{p, r\})=\mathcal{P}(p, r)$ is contained in $\mathcal{P}(p, s) \subseteq \mathcal{C}_{d_{\mathbf{P}^{-}}}$join $\left(q, \mathcal{C}_{d_{\mathbf{P}}}-h u l l(\{p, s\})\right)$ and, hence, $d_{\mathbf{P}}$ is stiff.

Finally, we have to show that $d_{\mathbf{P}}$ is extensible. To see this let $\mathcal{P}$ be a shortest path from $p$ to $q$ in $\mathbf{P}$. Without loss of generality assume that $p$ and $q$ do not belong to the boundary of $\mathbf{P}$. Then, we can extend the first line segment $s_{1}$ and last line segment $s_{2}$ of $\mathcal{P}$ until they intersect the boundary of $\mathbf{P}$ in two points, say $p_{1}$ and $q_{1}$ (see Figure $3 \mathrm{~b}$ ). We denote the extension of $s_{i}$ by $s_{i}^{\prime}$, for $i=1,2$. Assume that $s_{1}^{\prime}=\overline{p_{1} p_{2}}$ with $p_{2}$ on $\mathcal{P}(p, q)$. Then, $p$ belongs to $\mathcal{P}\left(p_{1}, p_{2}\right)$ and $p_{2}$ belongs to $\mathcal{P}(p, q)$. Hence, by Lemma 5.1 $\mathcal{P}\left(p_{1}, q\right)$ contains $\mathcal{P}(p, q)$. With a similar argument we obtain that $\mathcal{P}\left(p_{1}, q_{1}\right)$ contains $\mathcal{P}\left(p_{1}, q\right)$, and, thus, the first condition of extensibility is satisfied. The second condition follows trivially since we consider the inner metric of 
$\mathbf{P}$ and, hence, we can connect any two points with a $d_{\mathbf{P}^{-}}$straight path in $\mathbf{P}$.

\section{$5.2\left\{0^{\circ}, 90^{\circ}\right\}^{s}$-convexity}

Another interesting example are the semi-convex spaces generated by the notion of $\left\{0^{\circ}, 90^{\circ}\right\}^{s}$-convexity which is also called staircase convexity and well investigated [Bre94a, Bre94b, CR89, MRS88, Raw87, RW88]. We say that a subset $\mathrm{X}$ of $\mathbb{I E}^{2}$ is $\left\{0^{\circ}, 90^{\circ}\right\}$-convex if its intersection with every horizontal or vertical line is connected. A $\left\{0^{\circ}, 90^{\circ}\right\}$-convex path is called a $\left\{0^{\circ}, 90^{\circ}\right\}$ stairsegment. The connected components of $\left\{0^{\circ}, 90^{\circ}\right\}$-convex sets have the interesting property that each point can be connected to any other point by an $\left\{0^{\circ}, 90^{\circ}\right\}$-stairsegment that is completely contained in the component ([Raw87, Theorem 5.5.2]). We call a set that satisfies this last condition $\left\{0^{\circ}, 90^{\circ}\right\}^{s}$-convex. In order to make use of the results of Section 4 we note that the $L_{1}$-straight paths in the plane are just the $\left\{0^{\circ}, 90^{\circ}\right\}$-stairsegments. It is our aim to show that the $\left\{0^{\circ}, 90^{\circ}\right\}^{s}$-kernel of a simple polygon $\mathbf{P}$ in the plane (the points in $\mathbf{P}$ that can see every other point in $\mathbf{P}$ via a $\left\{0^{\circ}, 90^{\circ}\right\}$ stairsegment) is $\left\{0^{\circ}, 90^{\circ}\right\}^{s}$-convex. Since a simple polygon is a simple domain, we have to show only that $L_{1}$ is straight, extensible, and stiff. Once we have done so we conclude from the Kernel Theorem and Theorem 4.3 that the $\left\{0^{\circ}, 90^{\circ}\right\}^{s}$-kernel of any subset of $\mathbf{P}$ is $\left\{0^{\circ}, 90^{\circ}\right\}^{s}$-convex. This, in particular, implies that the $\left\{0^{\circ}, 90^{\circ}\right\}^{s}$-kernel of $\mathbf{P}$ is $\left\{0^{\circ}, 90^{\circ}\right\}^{s}$-convex.

So it remains to show only that $L_{1}$ satisfies the three properties of Theorem 4.3. That $L_{1}$ is straight follows immediately from the fact that $L_{1}$ is induced by a norm. We now consider the stiffness of $L_{1}$. Let $p, q, r$, and $s$ be four points in the plane such that there is a $\left\{0^{\circ}, 90^{\circ}\right\}$-stairsegment $\mathcal{P}_{p r}$ from $p$ to $r$ that contains $q$, a $\left\{0^{\circ}, 90^{\circ}\right\}$-stairsegment $\mathcal{P}_{q s}$ from $q$ to $s$ that contains $r$ and a $\left\{0^{\circ}, 90^{\circ}\right\}$-stairsegment $\mathcal{P}_{p s}$ from $p$ to $s$ (see Figure 5). Without loss of generality assume that $r$ is to the right and above $p$. If $q$ is not on the same vertical or horizontal line segment as $r$, then $r$ is also to the right and above $q$ and, hence, any $\left\{0^{\circ}, 90^{\circ}\right\}$-stairsegment from $q$ to $r$ is from bottom left to top right. Therefore, $s$ cannot be below or to the left of $r$ and any $\left\{0^{\circ}, 90^{\circ}\right\}$-stairsegment from $p$ to $r$ gives rise to a $\left\{0^{\circ}, 90^{\circ}\right\}$-stairsegment from $p$ to $s$ by just adding the part of the $\left\{0^{\circ}, 90^{\circ}\right\}$-stairsegment from $q$ to $s$ which is after $r$.

Now assume that $q$ is on the same horizontal line segment as $r$ and that $s$ is below and to the right of $q$. Consider a $\left\{0^{\circ}, 90^{\circ}\right\}$-stairsegment $\mathcal{S}$ from $p$ to $r$. We have to show that $\mathcal{S}$ is contained in $\mathcal{C}_{L_{1}}$ join $\left(q, \mathcal{C}_{L_{1}}-h u l l(\{p, s\})\right)$. Let

$x$ be the last intersection point of $\mathcal{S}$ with $\mathcal{P}_{s p} * \mathcal{P}_{p r}$ on $\mathcal{S}$. Clearly, the part of 


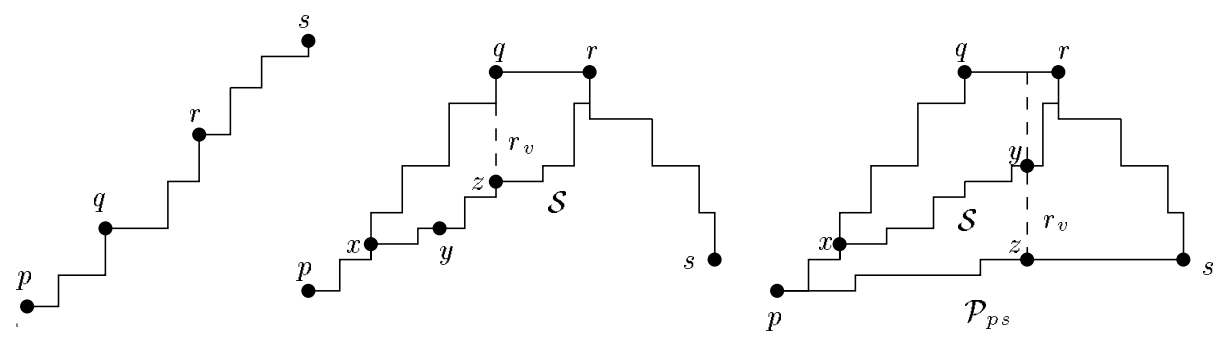

Figure 5: Proving the stiffness of $L_{1}$.

$\mathcal{S}$ from $p$ to $x$ belongs to $\mathcal{C}_{L_{1}}-\operatorname{hull}(\{p, q\}) \cup \mathcal{C}_{L_{1}}-h u l l(\{p, s\})$. Let $y$ be some point on $\mathcal{S}$ after $x$. There are two cases to be considered. If $y$ is to the left of $q$, then we consider the vertical ray $r_{v}$ downward from $q$. Since $r$ is above $s$ and to the right of $q$ and there are no intersection points of $\mathcal{S}$ with $\mathcal{P}_{p s}$ after $q$, the first path $r_{v}$ intersects is $\mathcal{S}$. Let $z$ be the intersection point. The boundary of $\mathbf{P}$ cannot cross the line segment $l$ from $q$ to $z$, since otherwise the intersection point would be enclosed by the curve $\mathcal{P}_{p r} * \mathcal{P}_{r s} * \mathcal{P}_{s q}$ which contradicts the fact that $\mathcal{P}_{p r} * \mathcal{P}_{r s} * \mathcal{P}_{s q}$ does not intersect the exterior of $\mathbf{P}$ and that $\mathbf{P}$ is simply connected. Since the part $\mathcal{S}_{1}$ of $\mathcal{S}$ from $p$ to $z$ stays below and to the left of $z, \mathcal{S}_{1} * l$ is a $\left\{0^{\circ}, 90^{\circ}\right\}$-stairsegment from $p$ to $q$. Hence, $y \in \mathcal{S}_{1} * l \subseteq \mathcal{C}_{L_{1}}-h u l l(\{p, q\})$.

Now suppose that $y$ is to the right of $q$. Since $y$ is on a $\left\{0^{\circ}, 90^{\circ}\right\}$ stairsegment from $p$ to $r, y$ is to the left of $r$. Hence, there is a point $z$ on the horizontal line segment from $q$ to $r$ that has the same $x$-coordinate as $y$. We denote the line segment from $q$ to $z$ by $l_{1}$. Consider the vertical ray $r_{v}$ downward starting in $z$. By the same argument as above we have that $r_{v}$ intersects $\mathcal{S}$ in $y$ before it intersects $\mathcal{P}_{p s}$ and that the line segment $l_{2}$ from $z$ to $\mathcal{P}_{p s} \cap r_{v}$ does not cross the boundary of $\mathbf{P}$. Hence, $y$ belongs to the path consisting of $l_{1} * l_{2}$ which is part of $\mathcal{C}_{L_{1}}-$ join $\left(q, \mathcal{C}_{L_{1}}-\operatorname{hull}(\{p, s\})\right)$. This completes the proof.

We now show the extensibility of $L_{1}$. Let $\mathcal{S}$ be a $d$-straight path from $p$ to $q$ in $\mathbf{P}$. Without loss of generality assume that $q$ is to the right and above $p$. We extend $\mathcal{S}$ by two horizontal line segments, one to the left of $p$ and one to the right of $q$ that intersect the boundary of $\mathbf{P}$. It is easily seen that $\mathcal{S}$ stays $\left\{0^{\circ}, 90^{\circ}\right\}$-convex if we append the line segments.

The last property we have to show is that all the points in a $L_{1}$-triangle 
T can be connected to the corner points with a $L_{1}$-straight path that is completely contained in $\mathbf{T}$. In order to do so, we make use of the following powerful result by R. Menger [Rin61, p. 148,17.5].

Theorem 5.2 If $\mathrm{X}$ is metrically complete and, for all points $p, q \in \mathrm{X}$, there exists a third point $r \in \mathbf{X}$ with $d(p, r)+d(r, q)=d(p, q)$, then any two points can be connected by a d-straight path contained in $\mathrm{X}$.

Hence, consider a $L_{1}$-triangle $\mathbf{T}$ which is bounded by the curves $\mathcal{P}_{p q}, \mathcal{P}_{q r}$, and $\mathcal{P}_{\text {rp }}$ and two points $x$ and $y$ in $\mathbf{T}$. We want to show that there is at least one point $z$ in $\mathbf{T}$ that lies between $x$ and $y$; that is, $z$ satisfies the equation $L_{1}(x, z)+L_{1}(z, y)=L_{1}(x, y)$. First, if $x$ is an inner point of $\mathbf{T}$, then since the set of all points that lie between $x$ and $y$ is just the rectangle $\mathbf{R}$ spanned by $x$ and $y$ and there is some neighbourhood $N$ of $x$ that is contained in $\mathbf{T}$, $N \cap \mathbf{R}$ contains at least one point different from $x$ that lies between $x$ and $y$. Hence, we can assume that $x$ and $y$ are on $\mathcal{P}_{p q} * \mathcal{P}_{q r} * \mathcal{P}_{r p}$. Since the paths are $L_{1}$-straight, we can assume that $x$ and $y$ are on two different paths, say $\mathcal{P}_{p q}$ and $\mathcal{P}_{p r}$. Without loss of generality assume that $y$ is above and to the right of $x$ and that $x$ is no corner point. Since $y$ is on the same side of $\mathcal{P}_{p q}$ as $\operatorname{int}(\mathbf{T})$, there is some part of the horizontal ray to the right starting in $x$ that belongs to $\mathbf{T}$.

Hence, for every two points $x$ and $y$ in $\mathbf{T}$, there exists a point that lies between them. By Theorem 5.2 we then have that any two points in $\mathbf{T}$ can be connected by a $L_{1}$-straight path.

\section{Conclusions}

We have introduced a definition of visibility in the abstract setting of semiconvex spaces and explored some of its consequences. Our main concern has been to characterize the semi-convex spaces that satisfy the Kernel Theorem. While we have been able to prove a very general relationship between skulls and kernels for arbitrary semi-convex spaces, the problem of finding necessary and sufficient conditions for semi-convex spaces, in which all kernels are convex, remains elusive. We feel that the conditions considered in this paper cannot capture the convexity of kernels and new concepts will have to be introduced to answer this question.

A second concern is the computational implications of the results. We showed that when the Kernel Theorem holds, it suffices to consider any minimal cover of skulls to find the kernel. For polygons in the plane this means that we have to consider at most $n$ skulls, where $n$ is the number 
of edges of the polygon; this observation holds although there may be an infinite number of skulls. There are, of course, more efficient algorithms to compute the kernel of a polygon in the plane, but it is doubtful whether the framework of semi-convex spaces allows the development of more efficient algorithms without making crucial use of the topological properties of the Euclidean plane or the $n$-dimensional Euclidean space.

\section{References}

[Bre82] M. Breen. Clear visibility and the dimension of kernels of starshaped sets. Proc. Am. Math. Soc., 85:414-418, 1982.

[Bre94a] M. Breen. A Krasnosel'skii theorem for staircase paths in orthogonal polygons. J. of Geometry, 51:22-30, 1994.

[Bre94b] M. Breen. An improved Krasnosel'skii-type theorem for orthogonal polygons which are starshaped via staircase paths. $J$. of Geometry, 51:31-35, 1994.

[CR89] J. Culberson and R. Reckhow. A Unified Approach to Orthogonal Polygon Covering Problems via Dent Diagrams. Technical Report TR 89-6, Dep. of Computing Science, Univ. of Alberta, 1989.

[Deg79] E. Degreef. Pasting and folding convexity spaces. Bull. de la Societé Math. du Belgique, XXXI(Fasc. II-Ser. B):215-230, 1979.

[DRS81] J. P. Doignon, J. R. Reay, and G. Sierksma. A Tverberg-type generalization of the Helly number of a convexity space. $J$. of Geometry, 16:117-125, 1981.

[Eck68] J. Eckhoff. Der Satz von Radon in konvexen Produktstrukturen I. Monatshefte für Math., 72:303-314, 1968.

[GHL*87] L. Guibas, J. Hershberger, D. Leven, M. Sharir, and R. E. Tarjan. Linear time algorithms for visibility and shortest path problems inside triangulated simple polygons. Algorithmica, 2:209233, 1987.

[GN90] L. P. Gewali and S. Ntafos. Minimum covers for grids and orthogonal polygons by periscope guards. In Jorge Urrutia, editor, Proc. 2nd Canadian Conf. in Computational Geometry, pages 358-361, Univ. of Ottawa, 1990.

[Ham63] P. C. Hammer. Extended topology: Domain finiteness. Indagationes Mathematicae, 25:200-212, 1963. 
[Jam82] R. E. Jamison-Waldner. A perspective on abstract convexity: classifying alignments by varieties. In D. C. Kay and M. Breen, editors, Convexity and Related Combinatorial Geometry, Proc. of the $2^{\text {nd }}$ Univ. of Oklahoma Conference, pages 113-150, 1982. Lecture Notes in Pure and Applied Mathematics, 76.

[Kei86] J. M. Keil. Minimally covering a horizontally convex polygon. In Proc. 2nd ACM Symp. on Computational Geometry, pages 43-51, 1986.

[KW71] D. C. Kay and E. W. Womble. Axiomatic convexity theory and the relationship between the Carathéodory, Helly and Radon numbers. Pacific J. of Math., 38:471-485, 1971.

[Lev51] F. W. Levi. On Helly's theorem and the axioms of convexity. $J$. of the Indian Math. Society, 15:65-76, 1951.

[LP81] W. Lipski and C. H. Papadimitriou. A fast algorithm for testing for safety and detecting deadlocks in locked transaction systems. J. of Algorithms, 2:211-226, 1981.

[MOW87] J. I. Munro, M. Overmars, and D. Wood. Variations on visibility. In Proc. 3rd ACM Symp. on Computational Geometry, pages 291-299, 1987.

[MRS88] R. Motwani, A. Raghunathan, and H. Saran. Covering orthogonal polygons with star polygons: The perfect graph approach. In Proc. 4th ACM Symp. on Computational Geometry, pages 211$223,1988$.

[OW88] M. Overmars and D. Wood. On rectangular visibility. J. of Algorithms, 9:372-390, 1988.

[Raw87] G. J. E. Rawlins. Explorations in Restricted-Orientation Geometry. PhD thesis, University of Waterloo, 1987.

[Rin61] W. Rinow. Die innere Geometrie der metrischen Räume. Volume 105 of Die Grundlehren der math. Wissenschaften in Einzeldarstellungen, Springer Verlag, 1961.

[Rin75] W. Rinow. Topologie. Volume 79 of Hochschulbücher für Mathematik, VEB Deutscher Verlag der Wissenschaften, 1975.

[RW88] G. J. E. Rawlins and D. Wood. Ortho-convexity and its generalizations. In G. Toussaint, editor, Computational Morphology, pages 137-152, Elsevier, 1988.

[RW89] G. J. E. Rawlins and D. Wood. A decomposition theorem for convexity spaces. J. of Geometry, 36:143-159, 1989. 
[Sie77] G. Sierksma. Relationships between Carathéodory, Helly, Radon and Exchange numbers of convexity spaces. Nieuw Archief voor Wiskunde, XXV:115-132, 1977.

[Sie81] G. Sierksma. Convexity on union of sets. Composito Mathematica, 42:391-400, 1981.

[Sie82] G. Sierksma. Radon partitions. Archiv der Mathematik, 39:568$576,1982$.

[Sie84] G. Sierksma. Extending a convexity space to an aligned space. Indagationes Mathematicae, 46:429-435, 1984.

[SW84] E. Soisalon-Soininen and D. Wood. Optimal algorithms to compute the closure of a set of iso-rectangles. J. of Algorithms, 5:199$214,1984$.

[Tor67] F. A. Toranzos. Radial functions of convex and starshaped sets. Am. Math. Monthly, 74:278-280, 1967.

[Tor88] F. A. Toranzos. Critical visibility and outward rays. J. of Geometry, 33:155-167, 1988.

[Tou88] G. Toussaint. A geodesic Helly-type theorem. In G. Toussaint, editor, Snapshots of Computational and Discrete Geometry, pages 135-138, McGill Univ., 1988. Technical Report SOCS88.11 .

[Val65] F. A. Valentine. Local convexity and $L_{n}$ sets. Proc. Am. Math. Soc., 16:1305-1310, 1965.

[vdV93] M. van de Vel. Theory of Convex Structures. Elsevier, Amsterdam, 1993. 